\title{
HP2 survey
}

\section{The California Molecular Cloud: A sleeping giant revisited ${ }^{\star}, \star \star$}

\author{
Charles J. Lada ${ }^{1}$, John A. Lewis ${ }^{1}$, Marco Lombardi ${ }^{1,2}$, and João Alves ${ }^{3}$ \\ 1 Harvard-Smithsonian Center for Astrophysics, Mail Stop 72, 60 Garden Street, Cambridge, MA 02138, USA \\ e-mail: clada@cfa.harvard.edu \\ 2 University of Milan, Department of Physics, via Celoria 16, 20133 Milan, Italy \\ ${ }^{3}$ University of Vienna, Türkenschanzstrasse 17, 1180 Vienna, Austria
}

Received 22 May 2017 / Accepted 3 August 2017

\begin{abstract}
We present new high resolution and dynamic range dust column density and temperature maps of the California Molecular Cloud derived from a combination of Planck and Herschel dust-emission maps, and 2MASS NIR dust-extinction maps. We used these data to determine the ratio of the $2.2 \mu \mathrm{m}$ extinction coefficient to the $850 \mu \mathrm{m}$ opacity and found the value to be close to that found in similar studies of the Orion B and Perseus clouds but higher than that characterizing the Orion A cloud, indicating that variations in the fundamental optical properties of dust may exist between local clouds. We show that over a wide range of extinction, the column density probability distribution function (pdf) of the cloud can be well described by a simple power law (i.e., $\mathrm{PDF}_{N} \propto A_{K}^{-\mathrm{n}}$ ) with an index $(n=4.0 \pm 0.1)$ that represents a steeper decline with $A_{K}$ than found $(n \approx 3)$ in similar studies of the Orion and Perseus clouds. Using only the protostellar population of the cloud and our extinction maps we investigate the Schmidt relation, that is, the relation between the protostellar surface density, $\Sigma_{*}$, and extinction, $A_{K}$, within the cloud. We show that $\Sigma_{*}$ is directly proportional to the ratio of the protostellar and cloud pdfs, i.e., $\operatorname{PDF}_{*}\left(A_{K}\right) / \operatorname{PDF}_{N}\left(A_{K}\right)$. We use the cumulative distribution of protostars to infer the functional forms for both $\Sigma_{*}$ and $\mathrm{PDF}_{*}$. We find that $\Sigma_{*}$ is best described by two power-law functions. At extinctions $A_{K} \lesssim 2.5 \mathrm{mag}, \Sigma_{*} \propto A_{K}^{\beta}$ with $\beta=3.3$ while at higher extinctions $\beta=2.5$, both values steeper than those $(\approx 2)$ found in other local giant molecular clouds (GMCs). We find that $\mathrm{PDF}_{*}$ is a declining function of extinction also best described by two power-laws whose behavior mirrors that of $\Sigma_{*}$. Our observations suggest that variations both in the slope of the Schmidt relation and in the sizes of the protostellar populations between GMCs are largely driven by variations in the slope, $n$, of $\operatorname{PDF}_{N}\left(A_{K}\right)$. This confirms earlier studies suggesting that cloud structure plays a major role in setting the global star formation rates in GMCs
\end{abstract}

Key words. stars: formation - dust, extinction - ISM: clouds - ISM: individual objects: California Molecular Cloud

\section{Introduction}

Located within the confines of the Perseus constellation, the California Molecular Cloud (CMC) rivals the Orion A molecular cloud as the most massive giant molecular cloud (GMC) within $0.5 \mathrm{kpc}$ of the Sun. However, despite its large mass, the CMC is a sleeping giant, characterized by a star formation rate (SFR) that is an order of magnitude lower than that of the Orion A cloud (Lada et al. 2009). This fact along with its proximity make the $\mathrm{CMC}$ an ideal laboratory for investigating the physical process which regulates the global level of star formation in a cloud and sets the SFR. Near-infrared (NIR) extinction mapping provided the first complete maps of the dust column density structure of the CMC (Lada et al. 2009; Lombardi et al. 2010). Analysis of these observations and comparison with similar observations of the Orion A cloud suggested a direct connection between the level of star formation activity in the cloud and its structural properties. In particular, it was suggested that

* HP2 (Herschel-Planck-2MASS) survey is a continuation of the series originally entitled "Herschel-Planck dust opacity and column density maps" (Lombardi et al. 2014; Zari et al. 2016).

$\star \star$ The reduced Herschel and Planck map and the column density and temperature maps are available at the CDS via anonymous ftp to

cdsarc.u-strasbg. fr (130.79.128.5) or via

http://cdsarc.u-strasbg.fr/viz-bin/qcat?J/A+A/606/A100 the global SFR of the cloud was set by the amount of high extinction ( $A_{K} \gtrsim 1.0 \mathrm{mag}$ ) material it contained (Lada et al. 2009).

In a subsequent study Kainulainen et al. (2009) showed that it was generally the case that actively star forming clouds contained more high extinction material than did quiescent clouds. Shortly thereafter Lada et al. (2010) demonstrated that a linear correlation exists between the measured SFRs and the gas masses at high extinction $\left(A_{K} \gtrsim 0.8 \mathrm{mag}\right)$ for a nearly complete sample of molecular clouds within $0.5 \mathrm{kpc}$ of the sun. The connection between the SFR and high cloud surface densities was further strengthened by detailed studies of both local low mass and more distant high mass clouds (Heiderman et al. 2010; Evans et al. 2014; Vutisalchavakul et al. 2016). There is also some evidence that this relation between SFR and dense gas extends smoothly to scales of entire galaxies (Wu et al. 2005; Lada et al. 2012; Jackson et al. 2013). A further investigation of the Schmidt relation (Schmidt 1959) within individual local molecular clouds, including the CMC, reinforced the idea that cloud structure was an important, if not the key, factor in determining the global SFR and level of star formation activity in a molecular cloud (Lada et al. 2013).

In light of the above considerations it would be of great interest to examine in more detail the relationship between star formation and the highest extinction gas. However, this is not feasible using existing NIR extinction maps because such maps 
are severely limited in their ability to probe the highest extinction regions, due to the lack of background stars that can be detected in the presence of such high dust opacity. More sensitive measurements of dust column density with higher dynamic range are required to investigate the nature of the star formation process at the high extinctions in which it occurs. With the dramatic improvements in the dynamic range of column density measurements provided by the Herschel mission, we are now in a position to re-examine the detailed structure of the CMC and its relation to star formation.

A significant fraction of the CMC was surveyed by Herschel (Harvey et al. 2013). The surveyed region contained about half the cloud mass but importantly all regions of significant star formation. Here we analyze both Herschel and Planck observations of the CMC using the methodology of Lombardi et al. (2014). This method combines the space observations with groundbased, NIR extinction maps to produce extinction-calibrated dust column density maps of a cloud. Such maps help mitigate the uncertainties in dust emission measurements of column densities and temperatures introduced by the well known degeneracy between these two physical quantities along a given line-of-sight. This is the third paper in a series applying this methodology to near-by clouds (Lombardi et al. 2014; Zari et al. 2016). The first two papers dealt with the more active star forming clouds Orion A, Orion B, and Perseus and demonstrated the power of the Herschel observations for probing high extinction material and providing dust column density maps of GMCs with high dynamic range and angular resolution. The results of those papers provide a very useful data set for comparison with the present study of the CMC and will help elucidate the nature star formation in this relatively quiescent GMC.

The layout of this paper is as follows. Section 2 describes the data used for this study. Section 3 reviews the methodology used to produce the dust column density and temperature maps. Section 4 presents the results and in Sect. 5 we discuss the implications. A summary of our primary conclusions is found in Sect. 6.

\section{Data}

The CMC was observed by the all-sky Planck observatory and by the Herschel Space Observatory as part of the "AurigaCalifornia" program (Harvey et al. 2013). The Herschel data we used consisted of observations obtained in parallel mode simultaneously using the PACS and SPIRE (Griffin et al. 2010) instruments. More details about the observational strategy can be found in Harvey et al. (2013) and André et al. (2010). We used the final data products of Planck (Planck Collaboration XI 2014). Following the methodology of Lombardi et al. (2014) and Zari et al. (2016), the Herschel data products were pre-processed using the Herschel interactive processing environment (HIPE; Ott 2010) version 15.0.1, with the latest version of the calibration files. The final maps were then produced using the HIPE level 2.5 of the SPIRE data, and the Unimaps data for PACS.

For the purposes of this study we use Herschel observations made in the PACS $160 \mu \mathrm{m}$ band, and the SPIRE $250 \mu \mathrm{m}$, $350 \mu \mathrm{m}$ and $500 \mu \mathrm{m}$ bands. However, prior to analyzing the data we multiplied each SPIRE band by an updated correction factor $C \equiv K_{4 \mathrm{e}} / K_{4 \mathrm{p}}$ (Zari et al. 2016) and then performed an absolute calibration of the Herschel fluxes using Planck maps. Next we convolved all Herschel data to the resolution of the SPIRE $500 \mu \mathrm{m}$ band, i.e., $F W H M_{500 \mu \mathrm{m}}=36$ arcsec.

A false color image of the reduced Herschel and Planck SPIRE band fluxes in the CMC region is shown in Fig. 1. The
Herschel observations cover the bulk of the molecular cloud in the central regions of the image. Here the three Herschel SPIRE passbands have been convolved to the $500 \mu \mathrm{m}$ resolution of 36 arcsec. The fluxes in the regions outside the Herschel survey area are the predicted Planck fluxes at the three SPIRE passbands and are characterized by a much lower angular resolution of 5 arcmin. We used the Planck/IRAS dust model (i.e., $T, \beta, \tau_{850}$; Planck Collaboration XI 2014) to derive the expected fluxes in the SPIRE passbands. The boundary between the two regions is clearly apparent in the image because of the differing angular resolutions of the two surveys. We note here that although these two regions are clearly demarked by their differing angular resolutions, the colors in the high resolution region match the colors of the rest of the image very well indicating that the absolute calibration of the Herschel observations is accurate.

Finally, we used the NIR extinction maps of the CMC from Lada et al. (2009) that were derived from the 2MASS all-sky survey with the NICEST technique (Lombardi 2009).

\section{Methodology}

We derived the dust column density and temperature maps of the cloud following the methodology developed by Lombardi et al. (2014). This method was previously used to construct similar maps of the Orion A and B clouds (Lombardi et al. 2014) as well as the Perseus cloud (Zari et al. 2016). Here we briefly outline the general procedure. For more details the reader can consult the Lombardi et al. paper.

\subsection{Physical model}

We consider the dust emission to be optically thin and describe the specific intensity at a frequency $v$ as a modified blackbody:

$I_{v}=B_{v}(T)\left[1-\mathrm{e}^{-\tau_{v}}\right] \simeq B_{v}(T) \tau_{v}$

where $\tau_{v}$ is the optical-depth at the frequency $v$ and $B_{v}(T)$ is the blackbody function at the temperature $T$ :

$B_{v}(T)=\frac{2 h v^{3}}{c^{2}} \frac{1}{\mathrm{e}^{h v / k T}-1}$.

Following standard practice, the frequency dependence of the optical depth $\tau_{v}$ can be written as

$\tau_{v}=\tau_{v_{0}}\left(\frac{v}{v_{0}}\right)^{\beta_{\mathrm{d}}}$,

where $v_{0}$ is an arbitrary reference frequency. We set $v_{0}=$ $353 \mathrm{GHz}$, corresponding to $\lambda=850 \mu \mathrm{m}$, and we indicate the corresponding optical depth as $\tau_{850}$. This is also the standard adopted by the Planck collaboration.

We note that when using this physical model we are implictly assuming that temperature gradients are negligible along the line-of-sight. This is an approximation because non-negligible gradients in the dust temperature are clearly observed in the plane of the sky in many regions of molecular clouds. Therefore, it is likely that in such regions temperature gradients exist along the line-of-sight as well. Because of the sensitive dependence of Eq. (1) on temperature, the temperatures derived from the observed fluxes using this equation will be biased somewhat toward higher values. This will ultimately result in slight underestimates in the opacities and corresponding column densities. Therefore $T$ in Eq. (1) should be considered an effective dust temperature for an observed dust column. 


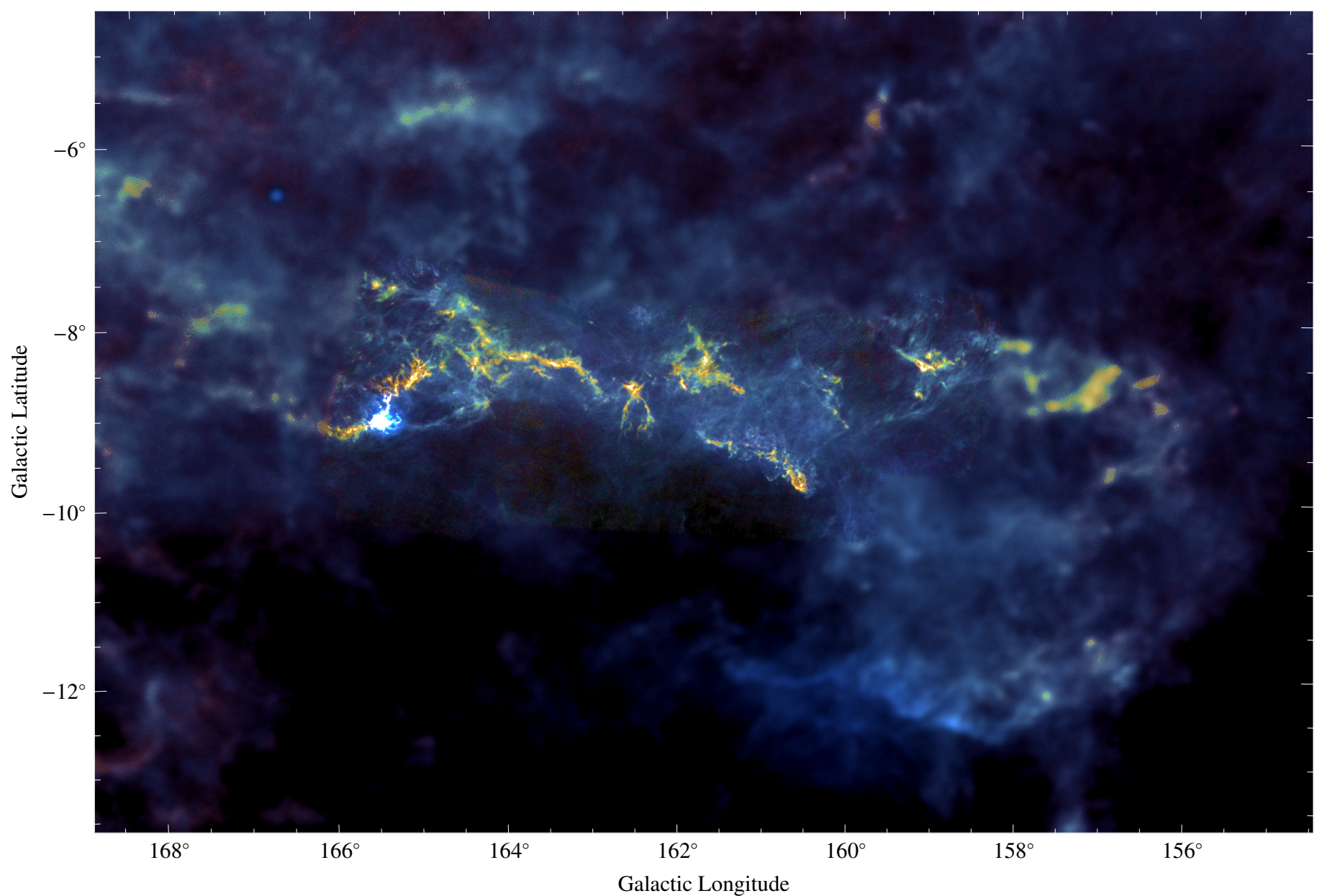

Fig. 1. False color composite image showing the $250 \mu \mathrm{m}$ (blue), $350 \mu \mathrm{m}$ (green), and $500 \mu \mathrm{m}$ (red) SPIRE fluxes for the California Cloud region derived from Herschel and Planck observations. In the regions outside the Herschel coverage a Planck/IRAS dust model was used to predict the corresponding SPIRE fluxes. The spatial boundary between the Herschel and the Planck regions is readily identified due to the differing angular resolutions of the two data sets.

\subsection{SED fit}

We can use our dust model to derive to derive the optical depth $\tau_{850}$, the effective dust temperature $T$, and the exponent $\beta_{\mathrm{d}}$ in a given map pixel by fitting the modified blackbody of Eq. (1) to the fluxes measured by Herschel for that pixel. We use the reduced observations in the PACS $100 \mu \mathrm{m}$ and $160 \mu \mathrm{m}$ bands, and the SPIRE $250 \mu \mathrm{m}, 350 \mu \mathrm{m}$ and $500 \mu \mathrm{m}$ bands to construct the spectral energy distribution (SED) at each map pixel. We fit the modified blackbody dust model to the SED using a simple $\chi^{2}$ minimization that takes into account the calibration errors (taken to be $15 \%$ in all bands). Because of the degeneracies present in the $\chi^{2}$ minimization, we fixed $\beta_{\mathrm{d}}$ and fit only for $\tau_{850}$ and $T$. For $\beta_{\mathrm{d}}$ we adopted local values computed by the Planck collaboration (Planck Collaboration XI 2014) on 35 arcmin scales across the entire sky.

\section{Results}

\subsection{Optical depth and temperature maps}

Figure 2 shows a combined optical depth and dust temperature map derived from the SED fits. Here the image intensity is proportional to the opacity and color scales with temperature with blue representing hot $(T>25 \mathrm{~K})$ dust and red representing cold $(T<15 \mathrm{~K})$ dust. The coldest regions of the cloud (red) have dust temperatures $\sim 14 \mathrm{~K}$ while the hottest blueish region is characterized by temperatures between $18-33 \mathrm{~K}$. These warmer temperatures are confined to the area around the embedded cluster associated with the $\mathrm{B}$ star $\mathrm{LkH} \alpha 101$ and the emission nebula NGC 1579. The dust in the bulk of the cloud is characterized by temperatures in the range $15-16 \mathrm{~K}$. Optical depths range from roughly $10^{-4}$ to $10^{-3}$ with the highest values appearing closely associated with the coldest dust. Separate dust temperature and opacity maps for the region are shown in Fig. 3.

\subsection{Converting opacities to extinctions and column densities}

We can derive extinctions and ultimately useful column densities for the cloud from direct comparison of the $\tau_{850}$ and $K$-band (NICEST) extinction $\left(A_{K}\right)$ maps. To make this comparison we considered only the Herschel observations and first smoothed the Herschel opacity map to the lower resolution ( 80 arcsec) of the extinction map. We empirically determined that between $0 \leq \tau_{850} \leq 2 \times 10^{-4}$ the relation between $\tau_{850}$ and $A_{K}$ is linear, that is:

$A_{K}(\mathrm{NIR})=\gamma \tau_{850}+\delta$.

Note that since $\tau_{v}=\kappa_{v} \Sigma_{\text {dust }}$, where, $\Sigma_{\text {dust }}$ is the dust-column density and $\kappa_{850}$ is the opacity coefficient (at $850 \mu \mathrm{m}$ ), the slope $\gamma$ is proportional to the ratio of $\kappa_{850}$ to $C_{2.2}$, the extinction coefficient 


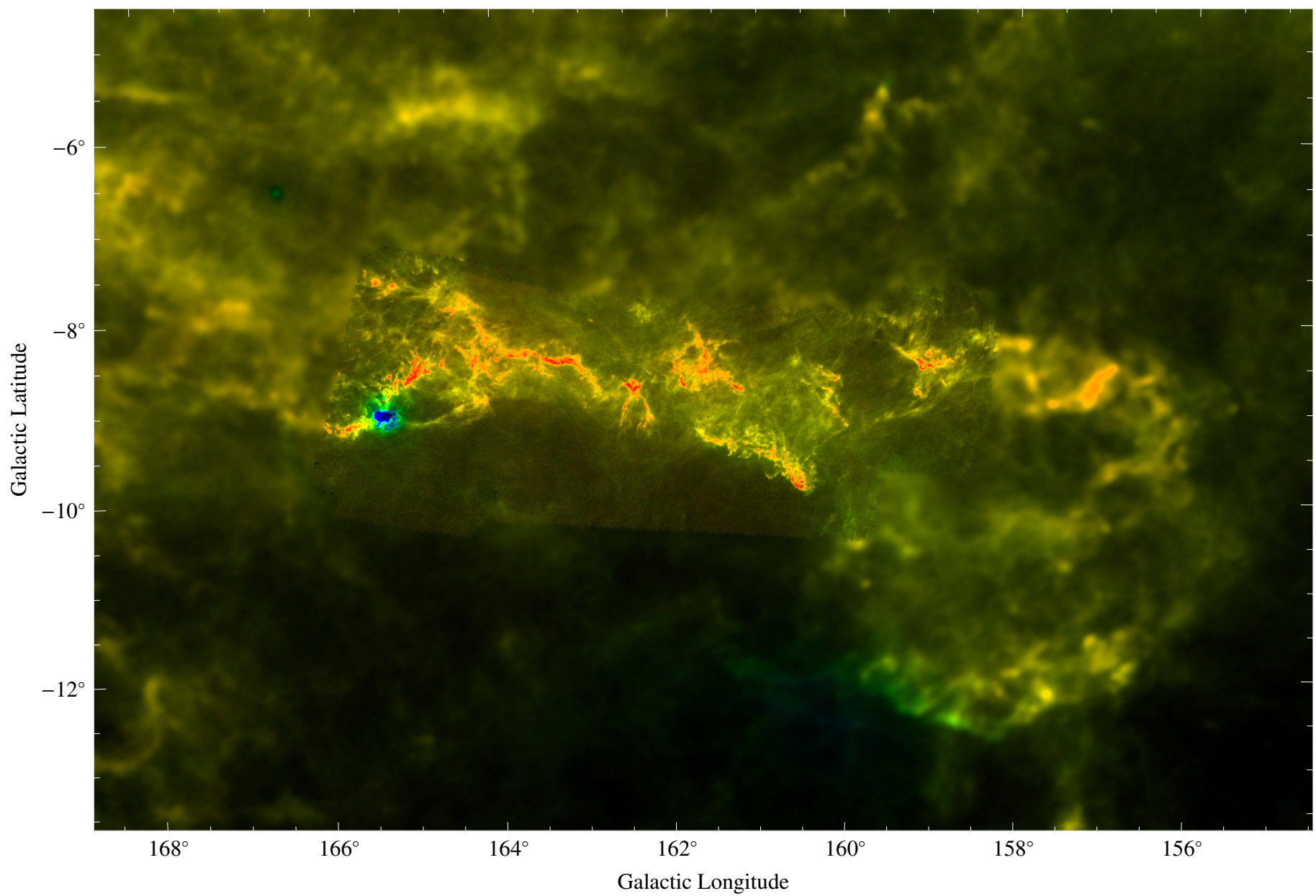

Fig. 2. False color image showing the optical depth-dust temperature map for the California Molecular Cloud. The image shows optical depth as intensity and temperature as color with blue (red) corresponding to high (low) dust temperature. See text.
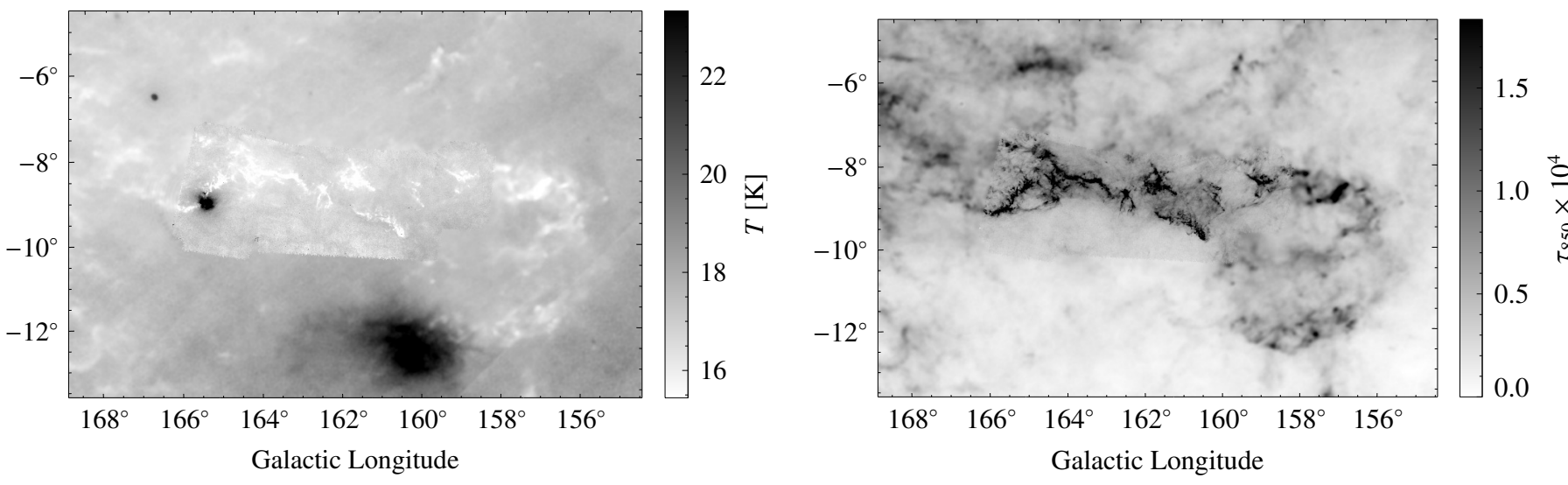

Fig. 3. Gray scale images showing the separate maps of dust temperature, $T_{\mathrm{D}}(\mathrm{eff})$, (left) and optical depth, $\tau_{850}$, (right) for the California Molecular Cloud. The large dark region of high dust temperature at $l \approx 160^{\circ}$ is the famous California Nebula, an HII region ionized by a runaway $\mathrm{O}$ star not associated with the California Molecular Cloud. The smaller, more compact hot region at $l \approx 165.5^{\circ}$ is NGC 1579 and it is excited by the only embedded cluster in the cloud. Neither region appears noticeable on the optical depth map, illustrating the low opacities of the dust within the two HII regions.

at $2.2 \mu \mathrm{m}$. This follows from:

$A_{K}=-2.5 \log _{10}\left(\frac{I_{\text {obs }}}{I_{\text {true }}}\right)=\left(2.5 \log _{10} \mathrm{e}\right) C_{2.2} \Sigma_{\text {dust }}$.

Thus, to first approximation, $\gamma \simeq 1.0857 C_{2.2} / \kappa_{850}$. The coefficient $\delta$ in Eq. (4) absorbs calibration and systematic uncertainties that arise in both the Herschel and extinction measurements.
From fitting the linear relationship of Eq. (4) to the data the following values for the two parameters were derived:

$\left\{\begin{array}{l}\gamma=3593 \pm 75 \mathrm{mag} \\ \delta=-0.110 \pm 0.006 \mathrm{mag} .\end{array}\right.$

The data and fit are displayed in Fig. 4. The same figure also shows the predicted $3 \sigma$ boundaries around the fit of Eq. (4). 


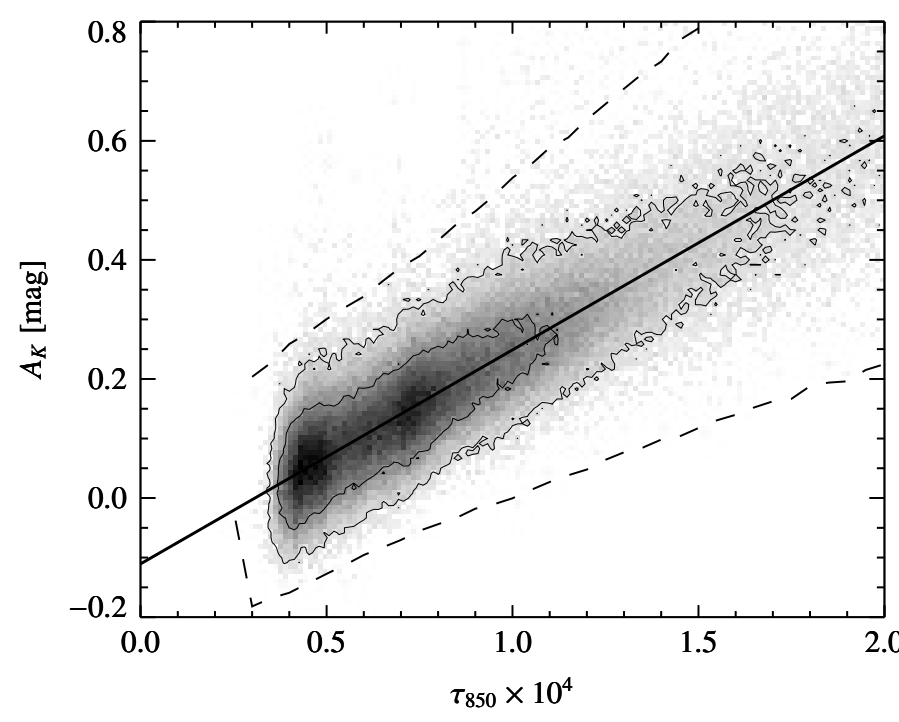

Fig. 4. Relationship between Herschel $\tau_{850}$ and NIR extinction in the CMC. The best linear fit, used for calibrating the opacities, is shown as a black line. Dashed lines indicate the expected $3 \sigma$ boundaries as calculated from direct error propagation in the extinction map. The contours enclose $68 \%$ and $95 \%$ of the points in the region, respectively. See text.

These boundaries were estimated from the statistical error on the extinction map alone (i.e., errors in the optical-depth were ignored). That the data points are predominately found to lie within $3 \sigma$ boundaries indicates that fit is accurate and confirms that the optical-depth map has a negligible relative error at the $80 \mathrm{arcsec}$ resolution of the extinction map. The value of $\gamma$ we found for the CMC is higher than that obtained in a similar study of the Orion A (2640; Lombardi et al. 2014) cloud, but similar to that derived for the Orion B cloud (3460; Lombardi et al. 2014) and the Perseus cloud (3931; Zari et al. 2016). As discussed in Lombardi et al. (2014) this suggests a possible variation in the optical properties of grains between local molecular clouds. The value of $|\delta|$ in the CMC is also higher than reported for the Orion and Perseus Clouds (i.e., $|\delta|=0.001-0.05$ ). However, as pointed out by Lombardi et al. (2010) the NIR extinction measurements used here underestimate the true values by a systematic offset of -0.04 mag due the presence of an unrelated screen of foreground extinction that contaminated the 2MASS control fields used for this area of the sky. Once adjusted for that systematic error the value of $\delta$ is about $-0.06 \mathrm{mag}$, comparable to those derived for the other regions.

For higher opacities the slope of the relation between $A_{K}$ and $\tau_{850}$ flattens and the relation becomes nonlinear. This departure from linearity and gradual flattening of the slope at higher extinctions is similar to that found previously for similar relations in Orion (Lombardi et al. 2014) and Perseus (Zari et al. 2016). It indicates that the infrared extinctions, $A_{K} \mathrm{~s}$, are more often underestimating than overestimating the dust column densities in regions of high opacity. This shift from linearity is the likely result of a breakdown in the extinction technique at high column densities due to the relative paucity of background stars within the indivdual 80 arcsec pixels of the 2MASS extinction map we used. This is confirmed by examining spatial maps of the differences, $A_{K}($ Herschel $)-A_{K}(2 \mathrm{MASS})$, which show the largest values confined to the regions of highest opacity, similar to what has been observed in the Orion (Lombardi et al. 2014) and Perseus clouds (Zari et al. 2016).

To construct a column density map of the region we convert the Herschel submillimeter opacities to extinctions using only the coefficient $\gamma$, that is we set:

$A_{K}($ Herschel $)=\gamma \tau_{850}$.

In using Eq. (7) we have ignored $\delta$ because of its relatively small magnitude and the presence of a systematic bias present in the NIR extinction measurements as discussed above. We applied Eq. (7) to the Herschel-Planck opacity map to derive extinctions in each map pixel. We find a dynamic range in extinction of $0.04 \leq A_{K} \leq 6.8 \mathrm{mag}$ across our entire map (i.e., Fig. 2). In Figs. 5-7 we present zoomed-in maps of the extinction derived from Eq. (7) for three interesting sub regions (A-C) of the CMC mapped by Herschel. These three maps were constructed at the higher spatial resolution (18 arcsec) of the SPIRE $250 \mu \mathrm{m}$ band following the methodology of Lombardi et al. (2014). Briefly, the SED of Eq. (1) was fit for $\tau_{250}$ in each SPIRE $250 \mu \mathrm{m}$ map pixel by using the flux in the $250 \mu \mathrm{m}$ band and fixing $T_{\mathrm{D}}$ to the value that was determined from the 36 arcsec map (i.e., Fig. 3) and fixing $\beta$ to the value derived by Planck as before. The value of $\tau_{850}$ was then derived from Eq. (3). In the high resolution maps our ability to resolve small, high opacity features was improved with the maximum measured infrared extinction increasing from 6.8 to 10.8 mag.

\subsection{Cloud Mass}

The extinctions derived from Eq. (7) can be converted to total (gas + dust) mass surface densities from:

$\Sigma_{\text {gas }}=\mu \alpha_{K} m_{\mathrm{p}}=183 A_{K} M_{\odot} \mathrm{pc}^{-2}$

here, $\mu=1.37$ is the mean molecular weight corrected for helium, $\alpha_{K}=1.67 \times 10^{22} \mathrm{~cm}^{-2} \mathrm{mag}^{-1}$ is the gas-to-dust ratio, $\left[N(\mathrm{HI})+2 N\left(\mathrm{H}_{2}\right)\right] / A_{K}$ (Bohlin et al. 1978; Rieke \& Lebofsky $1985)$ and $m_{\mathrm{p}}$ is the mass of a proton. The total mass of the cloud is then obtained from integrating over the (Herschel + Planck) surface area of the cloud:

$M_{\text {tot }}=\int \Sigma_{\text {gas }} \mathrm{d} S$.

To calculate the cloud mass we must first define the boundaries of the cloud. We consider the CMC to be entirely contained within a rectangular box on the sky given by $-13^{\circ}<b_{\mathrm{II}}<-6.5^{\circ}$ and $155^{\circ}<l_{\mathrm{II}}<167^{\circ}$. We define the physical cloud boundary to be given by the $A_{K}>0.2 \mathrm{mag}$ contour within the rectangular region. This region includes both the inner area of the cloud surveyed by Herschel as well as outer regions surveyed by Planck. We applied Eq. (7) to both data sets to derive the dust column densities across this expanse of the CMC. We then find $M_{\mathrm{tot}}=1.1 \times 10^{5} M_{\odot}$. The region surveyed by Herschel contains $5.52 \times 10^{4} M_{\odot}$ or roughly half the cloud mass at $A_{K}>0.2 \mathrm{mag}$.

\section{Discussion}

\subsection{Protostellar population and the star formation rate}

Knowledge of the protostellar population of a cloud is instrumental in determining the relation between the star formation rate (SFR) and structure of the cloud (e.g., Lombardi et al. 2013; Lada et al. 2013). Because of their relatively short lifetimes, the population of protostars in a cloud can be considered the instantaneous yield of the star formation process in that cloud. Indeed, the number of protostars $\left(N_{\mathrm{p} *}\right)$ is an excellent proxy for the instantaneous SFR since, to good approximation, $S F R=$ $m_{\mathrm{p} *} \tau_{\mathrm{p} *}^{-1} N_{\mathrm{p} *}$ where $\tau_{\mathrm{p} *}$ and $m_{\mathrm{p} *}$ are the typical lifetime and mass of 


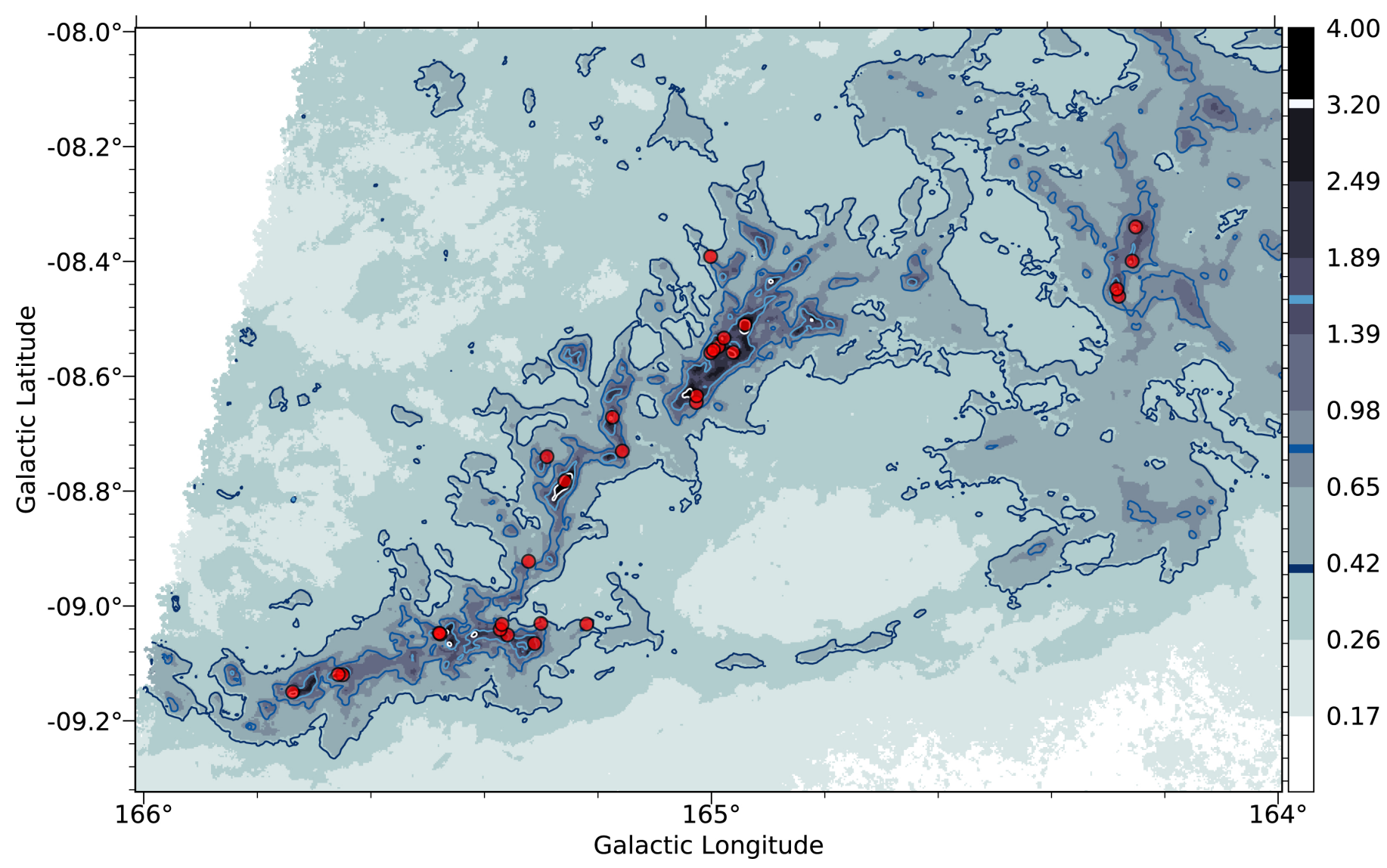

Fig. 5. Zoomed-in, high resolution map of infrared extinction $\left(A_{K}\right)$ derived from Herschel observations for southeastern region (A) of the California Molecular Cloud. Fiducial extinction contours as well as the position of known protostellar objects (filled red circles) are drawn on top of the gray scale map. The contour values are 0.4, 0.8, 1.6 and $3.2 \mathrm{mag}$ and are indicated by thick colored lines superposed on the adjacent scale bar. The angular resolution is 18 arcsec. See text.

a protostellar object. Moreover, because protostellar objects are most likely to be within or very close to their original birth sites, their surface densities are the most appropriate for comparison with those of the gas or dust. Therefore, we consider here only protostars (i.e., Class 0, Class I, and flat spectrum YSOs) in our determination of the cloud SFR.

IRAS observations provided the first census of young stellar objects which displayed characteristics of potential protostars in the California cloud. Seventeen candidate protostars were identified from IRAS observations by Lada et al. (2009). Deeper Herschel (Harvey et al. 2013) and Spitzer (Broekhoven-Fiene et al. 2014) observations resulted in independent and more complete YSO catalogs of the cloud including greatly improved source classifications. These catalogs increased the known number of YSOs to 60 and 166 objects, respectively, including both protostars and more evolved (Class II + III) stars.

Because the two catalogs were complied independently using slightly different criteria, there is some disagreement in source classifications between them. For this study we merged these two catalogs and re-examined the source classifications following procedure outlined by Lewis \& Lada (2016) and summarized in Appendix. Table A.1 presents the merged catalog of YSOs we adopted. We identify 43 protostars in the CMC.

The protostellar sources are largely confined to the portion of the cloud surveyed by Herschel. The locations of 42 of these protostellar sources are shown on the extinction maps in Figs. 57. These regions account for about $80 \%$ of the cloud mass mapped by Herschel and roughly $40 \%$ of the total cloud mass in the Herschel + Planck maps. Within the cloud boundary of $A_{K}>0.2$ mag the protostellar surface densities are not uniform with $\Sigma_{*}=0.2,0.03$ and $0.02 \mathrm{pc}^{-2}$ for regions A-C, respectively. Region $\mathrm{A}$ is considerably more active and efficient in forming stars than either B or C. Region A contains $\sim 70 \%$ of the protostars in the CMC while occupying only a small fraction (7\%) of the total (Herschel + Planck) cloud area and containing only a small fraction $(10 \%)$ of its total mass. This region also contains NGC 1579 (LK H $\alpha 101$ ) the only substantial embedded cluster in this massive cloud. In all three regions the distributions of protostars closely follows the distribution of high extinction gas.

\subsection{Cloud structure}

The area distribution function is defined as

$S\left(>A_{K}\right) \equiv \int_{A_{K}}^{\infty} \mathrm{d} S\left(A_{K}\right)$

where $\mathrm{d} S\left(A_{K}\right)$ is an element of cloud surface area at an extinction $A_{K}$. The area distribution function is a cumulative distribution that represents the total cloud area above a given threshold extinction as a function of $A_{K}$. The derivative of this function, $-S^{\prime}\left(>A_{K}\right)$ is proportional to the column density PDF of the cloud and both functions are useful descriptors of cloud structure. Figures 8 and 9 show the functions $S\left(>A_{K}\right)$ and $-S^{\prime}\left(>A_{K}\right)$ respectively. (We note that as plotted here the latter function corresponds to a linearly binned PDF and consequently its slope 


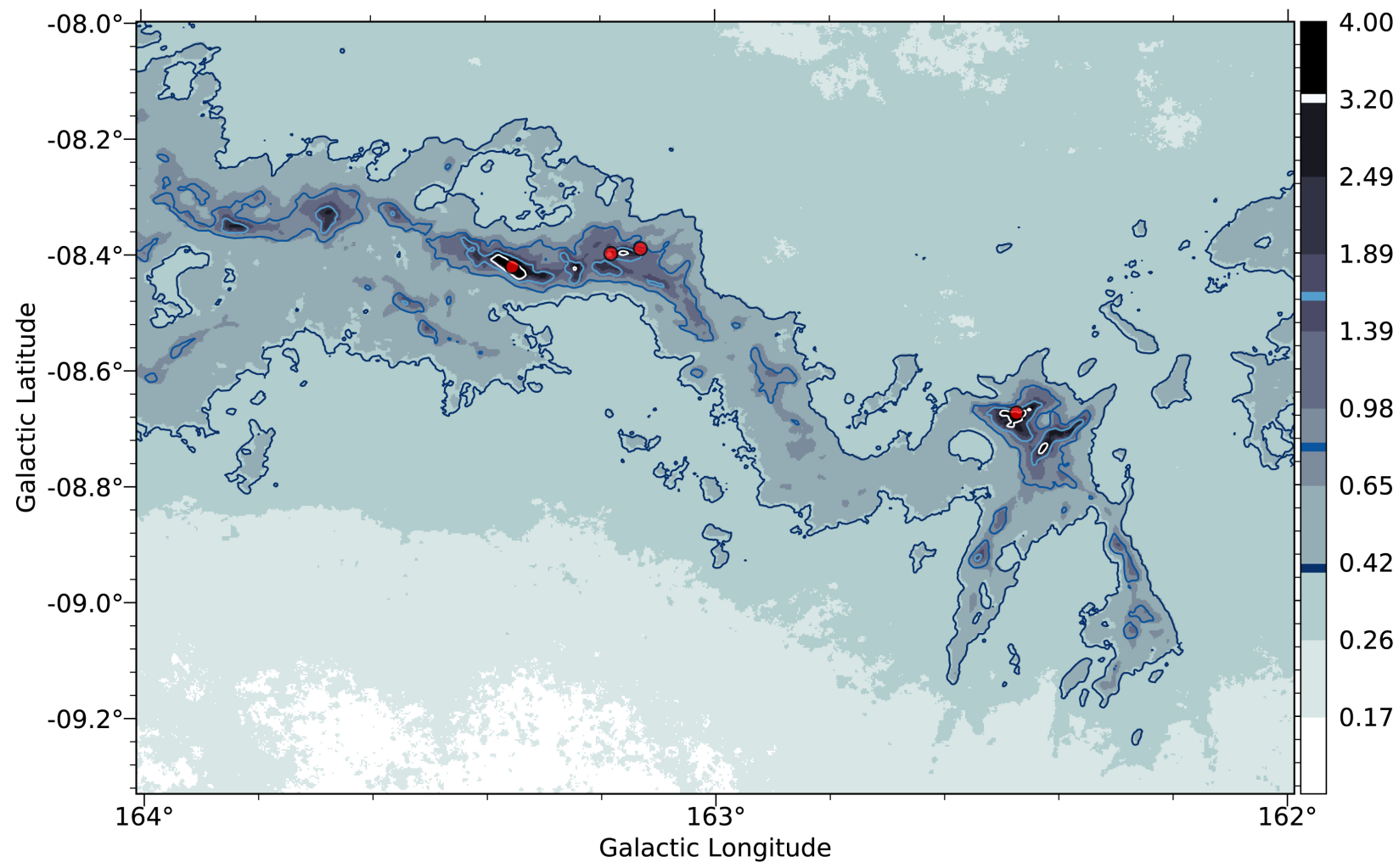

Fig. 6. Zoomed-in, high resolution map of infrared extinction $\left(A_{K}\right)$ derived from Herschel observations for central region (B) of the California Molecular Cloud. Otherwise same as Fig. 5.

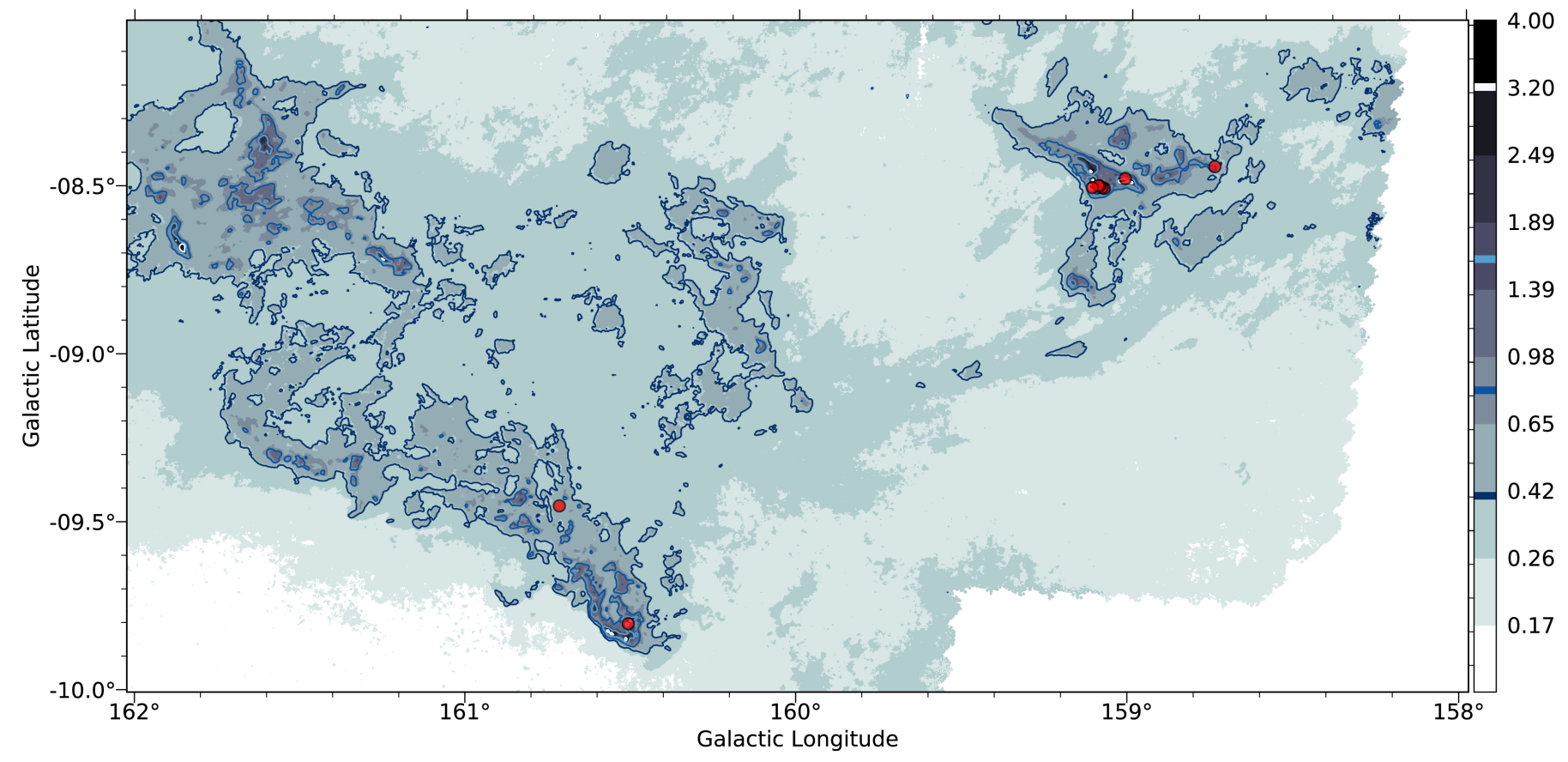

Fig. 7. Zoomed-in map of infrared extinction $\left(A_{K}\right)$ derived from Herschel observations for northwestern region (C) of the California Molecular Cloud. Otherwise same as Fig. 5.

will differ by -1 from that of a logarithmically binned PDF, such as the ones studied by Lombardi et al. 2015.) Although the Herschel observations clearly extend to significantly higher extinctions than the 2MASS observations, in the range where the two data sets overlap, the two functions, $S\left(>A_{K}\right)$ and $-S^{\prime}\left(>A_{K}\right)$ are in reasonable agreement with both data sets, confirming the result of our earlier study (Lada et al. 2013) that both $S\left(>A_{K}\right)$ and $-S^{\prime}\left(A_{K}\right)$ fall off relatively steeply with $A_{K}$. For example, with $-S^{\prime}\left(>A_{K}\right) \propto A_{K}^{-n}$ we find, from a formal fitting of the CMC data, that $n=4.0 \pm 0.1$, for $A_{K}>0.35$ mag. This is 


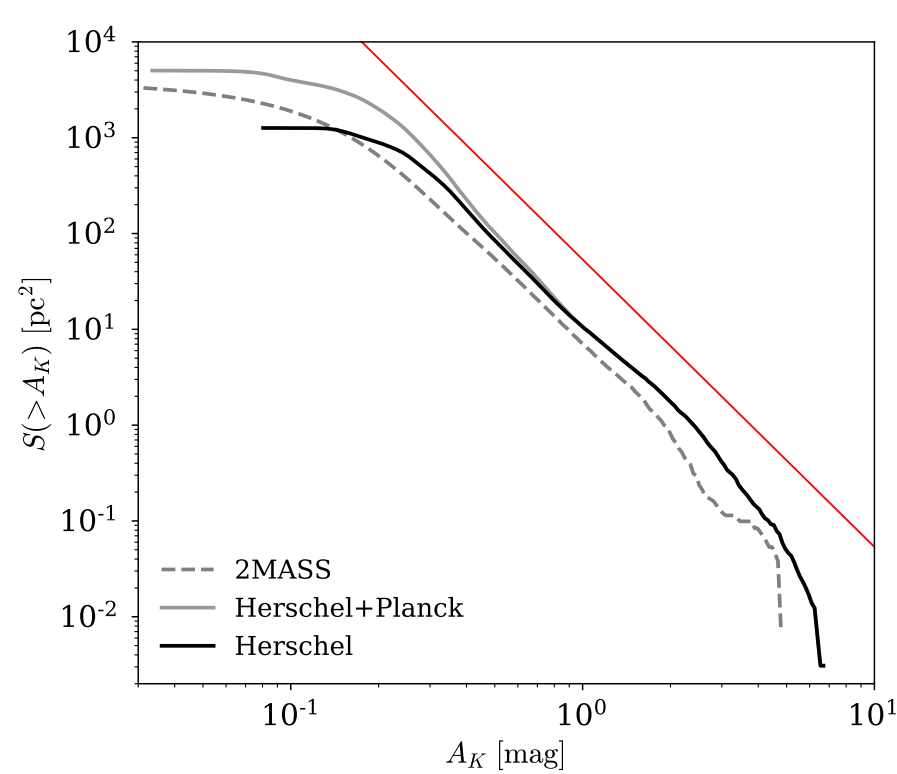

Fig. 8. Surface area distribution functions, $S\left(>A_{K}\right)$, for the California cloud. The solid red line represents a power law relation with a slope of -3 for comparison.

relatively steep compared to the value $(\approx 3)$ that describes the pdfs in Perseus and Orion. Similarly for $S\left(>A_{K}\right) \propto A_{K}^{-q}$, where $q=n-1=3.0$, the relation is relatively steep compared to that found $(\approx 2)$ in the active star forming clouds Orion A, Orion B, and Perseus that we have also studied with Herschel.

\subsection{The Schmidt relation}

In previous studies of the CMC Lada etal. (2013) and Harvey et al. (2013) showed that a Schmidt relation of the form $\Sigma_{*} \propto A_{K}^{\beta}$ existed for the cloud. Here $\Sigma_{*}$, the surface density of protostellar objects and $A_{K}$ are proxies for $\Sigma_{S F R}$ and $\Sigma_{\text {gas }}$, respectively in the usual Schmidt relation (Schmidt 1959). However, using different methodologies and extinction data as well as slightly differing YSO catalogs they derived different values (2 and 4 , respectively) for the index $\beta$. In this paper we revisit the Schmidt relation for the CMC using a Bayesian (MCMC) methodology similar to Lada et al. (2013) coupled with our extinction calibrated, Herschel 36 arcsec resolution dust column density maps and our revised catalog of protostellar objects for the cloud.

We assume that the protostellar surface density behaves according to a thresholded Schmidt relation:

$\Sigma_{*}\left(A_{K}\right)=\kappa A_{K}^{\beta} H\left(A_{K}-A_{K, 0}\right)$,

where $\Sigma_{*}$ is the protostellar surface density, $A_{K}$ is the extinction in magnitudes in the pixel on which a source lies ${ }^{1}$, $H(x)$ is a Heaviside function, $A_{K, 0}$ is the extinction threshold for star formation, and $\kappa$ is a normalization factor with units $\left(\operatorname{star~pc}^{-2} \mathrm{mag}^{-\beta}\right)$. We do not model the diffusion of protostars from their birth location as was done in Lada et al. (2013) and Lombardi et al. (2014). The diffusion, as previously measured, is at the sub-pixel level and is not degenerate with any parameter, so will not affect our determination of $\kappa$ or $\beta$. We take

\footnotetext{
1 It is important to note that here $A_{K}$ represents the total extinction along the line-of-sight to the protostar averaged over a $36 \operatorname{arcsec}$ or $0.08 \mathrm{pc}$ region and is not the pencil-beam extinction to the source itself.
}

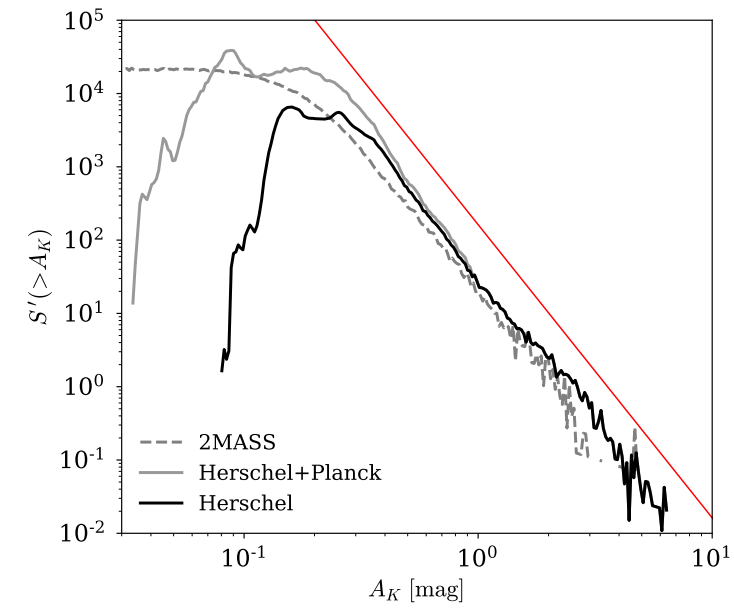

Fig. 9. Differential area function $-S^{\prime}\left(>A_{K}\right)$ which is proportional to the probability density distribution for column densities in the cloud. In this plot a lognormal distribution would appear as a Gaussian function whilst a power-law function would be a straight line. The red line shows the power-law $-S^{\prime}\left(>A_{K}\right) \propto A_{K}^{-4.0}$. See text.

the likelihood derived in Lombardi et al. (2013, their Eq. (7)), $\mathcal{L}\left(x_{n} \mid \theta\right)$, and estimate $\hat{\theta}=\left(\kappa, \beta, A_{K, 0}\right)$ using the affine-invariant MCMC package emcee (Foreman-Mackey et al. 2013), with the chains initialized to a Gaussian distribution around the result of a Nelder-Mead (amoeba) maximization of the likelihood.

From this analysis we determined the three credible intervals for the model in Eq. (8) to be $\beta=3.31 \pm 0.23, \kappa=$ $0.36 \pm 0.09$ stars pc $^{-2} \mathrm{mag}^{-3.31}$, and $A_{K, 0}=0.51 \mathrm{mag}$. In Fig. 10 we show the standard graphical representation of the power-law Schmidt relation we derived for the CMC plotted along with appropriately binned data for comparison. Visual inspection of the plot suggests that the fit and the model it is based on, i.e., Eq. (8), are more approximate than precise representations of the observations. In particular, the data in the highest extinction bins clearly deviate from the $\beta=3.31$ line. The departure of the highest extinction points from the fit suggests that a truncation may be present at high extinction. Such a truncation would likely result from the relative absence of high opacity material in the cloud as evidenced by the steep fall off of its PDF with $A_{K}$ (see Sect. 5.2 and Fig. 9).

The values of these posterior parameters differ significantly from those $\left(\beta \sim 2, \kappa \sim 2\right.$ and $\left.A_{K, 0} \sim 0.6 \mathrm{mag}\right)$ derived by Lada et al. (2013) using similar methodology. This difference could arise from the different extinction maps and protostellar catalogs used in our two studies. The difference in the protostellar catalogs employed was slight and likely not responsible for the differing results. Nonetheless, we performed our analysis on various subsets of the original Harvey et al. catalog, including a version that used all sources. We found the resulting posterior parameters to be the same within the errors and thus not particularly sensitive to the different catalogs used. We next performed our analysis using the 2MASS NIR extinction map instead of our Herschel map. In this case the analysis returned parameters that essentially reproduced the values derived by Lada et al. (2013). Closer inspection of the data showed that the extinctions associated with the individual protostars were almost always underestimated in the NIR map. This is not surprising since more than $80 \%$ of the protostars in the CMC are found at $A_{K}>1.0 \mathrm{mag}$ in the Herschel extinction map. At these high opacities the NIR derived extinctions are considerably less 


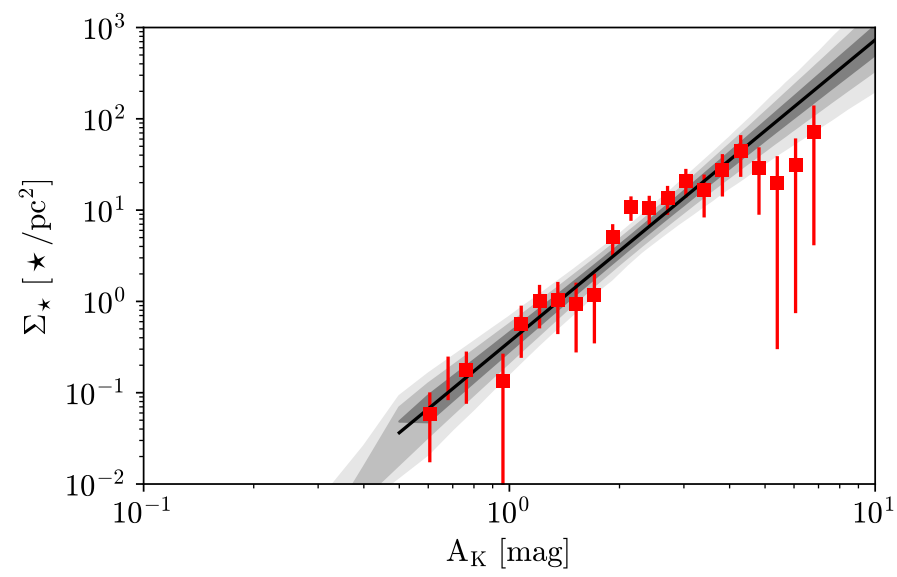

Fig. 10. Schmidt relation for protostellar objects in the CMC. The data is shown as red squares with corresponding uncertainties. The black line represents the power-law relation, $\Sigma_{\mathrm{YSO}}=\kappa A_{K}^{\beta}$ derived from the MCMC analysis with index $\beta=3.31$. The gray shaded regions represent the 1 , 2 , and $3 \sigma$ confidence levels for the plotted power-law relation. The data are fully sampled in logarithmic bins 0.1 dex wide equally spaced at intervals of $0.05 \mathrm{dex}$. See text.

reliable than our Herschel extinctions and moreover are expected to underestimate the true opacities due to the small numbers of detectable background stars present in the individual pixels. Our Herschel derived value of $\beta$ is closer to, but slightly less than, that ( 4) derived by Harvey et al. (2013) also using Herschel observations. Those authors employed a different methodology to estimate a value for $\beta$ : First, Harvey et al. (2013) produced surface density maps of dust $\left(A_{K}\right)$ and YSOs, both smoothed to a scale of $0.2 \mathrm{deg}$. They then made a ratio of the two maps and constructed the corresponding plot of $\Sigma_{*}$ vs. $A_{K}$ from that data. Harvey et al. (2013) quote only their value for $\beta$ derived from this plot and do not produce an estimate of its uncertainty so it is difficult to assess the significance of the difference between the two estimates. Because the value of $\beta$ derived using Herschel dust column densities by two different methods is higher than the value we previously derived using 2MASS extinctions, we adopt our improved estimate for this parameter as being the more faithful measure of its true value.

\subsection{The protostellar PDF}

We can gain some physical insight into the nature of the Schmidt relation and its connection to star formation by writing the relation in the following form:

$\Sigma_{*}\left(A_{K}\right)=\frac{\mathrm{d} N_{*}\left(A_{K}\right)}{\mathrm{d} S\left(A_{K}\right)}=\Sigma_{* 0} \times \frac{\operatorname{PDF}_{*}\left(A_{K}\right)}{\operatorname{PDF}_{N}\left(A_{K}\right)}$

here $\Sigma_{* 0}$ is a constant equal to the global protostellar surface density of the cloud, that is, the ratio of $N_{* \text { tot }}$, the total number of protostellar objects, to $S_{\text {tot }}$, the total cloud area. $\operatorname{PDF}_{*}\left(A_{K}\right)$ is the pdf of the protostellar population ${ }^{2}$ and $\operatorname{PDF}_{N}\left(A_{K}\right)$ is the cloud column density pdf. In this form we see that the Schmidt relation is proportional to the ratio of the protostellar and cloud pdfs. The column density pdf of a molecular cloud is a standard metric used to describe cloud structure. It has been shown to be well described by simple power-law functions of extinction (i.e., $\mathrm{PDF}_{N} \propto A_{K}^{-n}$; Lombardi et al. 2015). The protostellar pdf is not

\footnotetext{
$2 \operatorname{PDF}_{*}\left(A_{K}\right) \equiv \frac{1}{N_{* t o t}} \frac{\mathrm{d} N_{*}\left(A_{K}\right)}{\mathrm{d} A_{K}}$.
}

Table 1. Schmidt relation and PDF power-law indicies for GMCs studied with Herschel.

\begin{tabular}{ccccc}
\hline \hline GMC & $\beta$ & Reference & $n$ & Reference \\
\hline California & $3.31 \pm 0.23$ & 1 & 4.0 & 1 \\
Orion A & $1.99 \pm 0.05$ & 2 & 2.9 & 4 \\
Orion B & $2.16 \pm 0.10$ & 2 & 3.0 & 4 \\
Perseus & $2.4 \pm 0.6$ & 3 & 2.7 & 4 \\
\hline
\end{tabular}

References. (1) This paper; (2) Lombardi et al. (2014); (3) Zari et al. (2016); (4) Lombardi et al. (2015).

a well known distribution and to our knowledge has not been studied in the literature. Because $N_{*}\left(A_{K}\right)$ is proportional to the total instantaneous SFR at any extinction, the protostellar pdf is essentially the normalized measure of the SFR as a function of extinction. Consider that we can rewrite Eq. (9) to yield:

$\operatorname{PDF}_{*}\left(A_{K}\right)=\frac{\Sigma_{*}\left(A_{K}\right)}{\sum_{* 0}} \times \operatorname{PDF}_{N}\left(A_{K}\right)$.

If $\Sigma_{*}\left(A_{K}\right)$ and $\operatorname{PDF}_{N}\left(A_{K}\right)$ are both power-law functions of $A_{K}$, then $\mathrm{PDF}_{*}\left(A_{K}\right)$ must also be a power-law, i.e., $\operatorname{PDF}_{*}\left(A_{K}\right) \propto A_{K}^{p}$ where $p=\beta-n$.

In Table 1 we list the values of $\beta$ and $\mathrm{n}$ derived from similar analysis of local clouds with Herschel dust column densities. For all these clouds $n>\beta$ indicating that $\operatorname{PDF}_{*}\left(A_{K}\right)$ is a declining function of $A_{K}$ and predicting that the number of protostars will steeply decline with extinction, despite the rapid rise of the Schmidt relation with $A_{K}$. For the CMC we find $p=-0.69$ and for all four clouds $\langle\beta-n\rangle=-0.69 \pm 0.27$.

A protostellar pdf of the form $\operatorname{PDF}_{*}\left(A_{K}\right) \propto A_{K}^{-0.7}$ seems counterintuitive, in part because it clearly cannot describe the behavior of the actual $\operatorname{PDF}_{*}\left(A_{K}\right)$ at low extinctions. This is clear from Figs. 5-7, which show an almost complete absence of protostars in regions of low extinction (i.e., $A_{K} \lesssim 1.0 \mathrm{mag}$ ). Moreover, it also predicts that the number of protostars will sharply decline with increasing extinction and this appears in conflict with the fact that the protostellar positions seem to be correlated with the highest extinction regions in the maps of Figs. 5-7.

The relatively small numbers of protostars in the $\mathrm{CMC}$ coupled with the large dynamic range of extinction they sample make it difficult to directly determine a well sampled $\operatorname{PDF}_{*}\left(A_{K}\right)$ and test these predictions. However, we can gain further insight into the nature of the protostellar pdf by considering the normalized cumulative distribution of protostars:

$N_{*}\left(>A_{K}\right) / N_{* \text { tot }}=\int_{A_{K}}^{\infty} \operatorname{PDF}_{*}\left(A_{K}\right) \mathrm{d} A_{K}$.

This distribution can be considered the fractional yield of protostars as a result of the star formation process in the cloud. Figure 11 shows the normalized, cumulative distribution of protostars as a function of extinction observed in the CMC. As predicted above, the observed data points (filled symbols) indicate that the number of protostars in the cloud in fact does sharply drop off with increasing extinction. Similar results were found in the cumulative protostellar distributions in Orion A, Orion B, and Perseus. Moreover, the above analysis supports the hypothesis that, because $n>\beta$, the steep drop off of protostars at the highest extinctions is a direct result of the relative lack of such high extinction material in the cloud (Lada et al. 2013, and Fig. 9). Figure 11 also confirms that rather than increasing, the number of protostars at low extinctions becomes vanishingly 


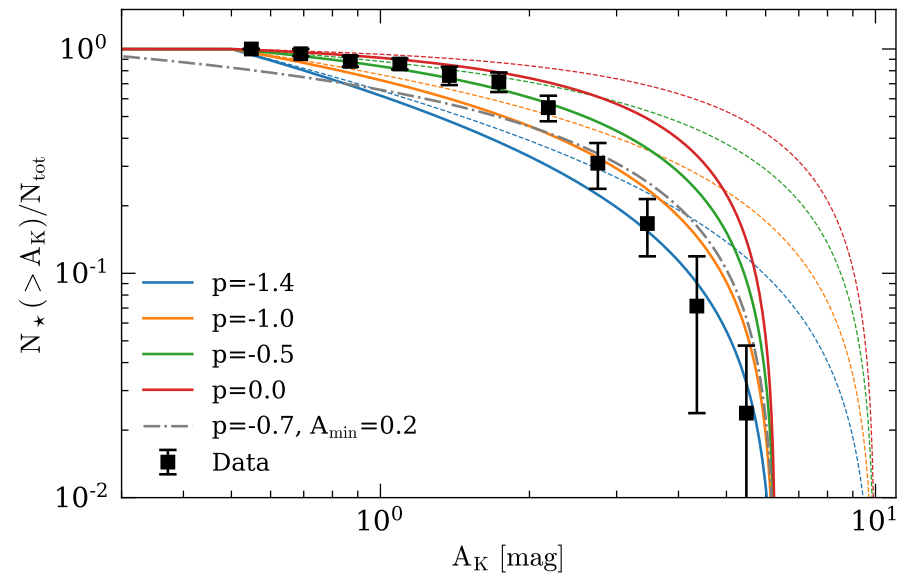

Fig. 11. Normalized cumulative distribution of protostellar objects with extinction in the CMC. The data are shown as filled squares with corresponding uncertainties. The continuous curves are the corresponding theoretical distributions expected for power-law protostellar pdfs i.e., $\mathrm{PDF}_{*}\left(A_{K}\right) \propto A_{K}^{p}$ and are truncated and normalized to 1.0 at $A_{K}=$ $0.5 \mathrm{mag}$. The solid curves correspond to $A_{\max }=6.3 \mathrm{mag}$ and lighter dashed traces correspond to $A_{\max }=10.0 \mathrm{mag}$. The dot-dash curve is normalized at $0.1 \mathrm{mag}$ to represent a cloud without an extinction threshold (see text).

small (i.e., $N_{*}\left(>A_{K}\right) / N_{\text {tot }}=1.0$, for $A_{K}<0.5 \mathrm{mag}$ ). Clearly the protostellar pdf cannot be described by a single power-law function that extends unabated to the lowest extinctions.

Using Eq. (11) with $\operatorname{PDF}_{*}\left(A_{K}\right) \propto A_{K}^{p}$, the normalized cumulative distribution of protostars can be written as:

$N\left(>A_{K}\right) / N_{\text {tot }}=(1+p) C_{p} \int_{A_{K}}^{A_{\max }} A_{K}^{p} \mathrm{~d} A_{K}$.

where $C_{p}$ is a normalization constant and $A_{\max }$ is the extinction measured in the highest extinction map pixel containing a protostar. In Fig. 11 we also plotted a series of theoretical cumulative distributions given by Eq. (12) for various values of the power-law index, $p$, of the protostellar pdf and two different values of the parameter $A_{\max }$. To be consistent with the observations of the CMC we have normalized the functions to equal 1.0 at $A_{K}=0.5 \mathrm{mag}$, effectively truncating the relations at that column density. For the solid set of curves we set $A_{\max }=6.3 \mathrm{mag}$, the value derived from the data. In the dashed set of curves we set $A_{\max }=10.0$ mag. The solid curves provide the best match to all the data.

Inspection of the figure confirms the notion that a single power-law $\operatorname{PDF}_{*}\left(A_{K}\right)$ is an inadequate description of the data. However, at high extinction $(\gtrsim 2.5 \mathrm{mag})$ the power-law with index $p=-1.4$ comes closest to matching the observations. In the range $0.5 \leq A_{K} \leq 2.0 \mathrm{mag}$, the data appear to best matched by the power-law with $p=-0.5$. From this simple comparison we can infer the general properties of the protostellar pdf in the CMC. At large extinctions $\operatorname{PDF}_{*}\left(A_{K}\right)$ does appear to fall off as a power-law with index $\approx-1.4$. The function departs from this power-law at intermediate extinctions and appears to follow a shallower power-law with $p \approx-0.5$ between 0.5 and $2.5 \mathrm{mag}$ and then truncates or falls off very rapidly with decreasing extinction. This latter regime forms a broad peak in the pdf and accounts for roughly $80 \%$ of the protostars in the cloud.

An instructive case is that of $p=0$. Here there is equal probability of finding a protostar at any given extinction. In this case the indicies of the Schmidt relation and the cloud pdf would be the same, i.e., $n=\beta$. This is clearly not the case in the observations. That $\beta$ is found to be positive suggests that the star formation process operates more efficiently in gas at high extinctions but since $\beta<n$, not efficiently enough to prevent a decrease in the relative number of protostars formed (and the SFR) at the highest extinctions.

The other set of curves plotted in Fig. 11 are cumulative distributions calculated from Eq. (12) with $A_{\max }=10.0 \mathrm{mag}$. These curves do not come close to matching the data and illustrate the sensitivity of the predicted distribution to the value of $A_{\max }$ and thus to $S^{\prime}\left(>A_{K}\right)$, the un-normalized cloud column density pdf. For example, in the $\mathrm{CMC}, A_{\max }$ is very close to the maximum observed extinction $(6.8 \mathrm{mag}$ ) in the 36 arcsec pixels. However, the $A_{\max }=10$ mag curves could correspond to a cloud with a $\operatorname{PDF}_{N}\left(A_{K}\right)$ that likely falls off more slowly with extinction and as a result contains considerably more high extinction material than the CMC. Comparison of these models with the data again illustrates the importance of cloud structure in controlling the star formation process in the cloud.

Finally, we plotted a curve (dashed-dot trace) for a powerlaw with index -0.7 that is normalized at an extinction of 0.2 mag. This illustrates the situation of a low extinction truncation that would correspond to the edge of a molecular cloud. This curve fails to match any of the observations. This reinforces the idea that there is a definite threshold for or truncation of the protostellar pdf at modest $\left(A_{K} \sim 0.5 \mathrm{mag}\right)$ extinctions in the CMC. This truncation or threshold may be the result of an additional steepening (i.e., $\beta>4$ ) of the Schmidt relation for $A_{K}<0.5 \mathrm{mag}$ in the CMC.

If the protostellar pdf cannot be described as a single powerlaw function then Eq. (9) requires that single power-law functions cannot describe the Schmidt relation and/or the cloud pdf. Inspection of Fig. 9 indicates that $\operatorname{PDF}_{N}\left(A_{K}\right)$ is very close to being a single power-law relation over a large range (i.e., $A_{K}>$ $0.2 \mathrm{mag}$ ) in extinction. On the other hand, as discussed earlier and shown in Fig. 10, the Schmidt relation in the CMC is not particularly well fit by a single power-law function over a similar extinction range. Indeed, since the power-law indicies of the three functions are related as $\beta=p+n$, a change in power-law slope $p$ would be directly reflected by a change in $\beta$ for a constant $n$. At high extinctions we would expect $\beta=4.0-1.4=2.6$ suggesting a flattening of the relation at high extinction as is seen in Fig. 10. At lower extinctions we would predict $\beta=4.0-0.5=3.5$, not far from the value of 3.3 that appears to fit the lower portion of the relation.

We can use the observed cumulative protostellar distribution function again to further constrain the Schmidt relation. Figure 12 plots the predicted distribution of protostellar objects obtained by numerically integrating:

$N_{*}\left(>A_{K}\right)=\int \Sigma_{*}\left(A_{K}\right) \mathrm{d} S=\int_{A_{K}}^{\infty} \Sigma_{*}\left(A_{K}\right)\left|-S\left(>A_{K}\right)\right| \mathrm{d} A_{K}$.

The integration is carried out over the observed differential area function with $\Sigma_{*} \propto A_{K}^{\beta}$ for differing values of $\beta$. The uncertainties are shown for the case of $\beta=3.31$ that we derived from the data assuming a model of a single power function. As observed in Figs. 10 and 11 a single power-law does not appear match the observations. Allthough the low extinction data are well matched by the nominal $\beta \approx 3.3$ curve, the high extinction data in Fig. 12 fall below the nominal $\beta=3.3$ curve and are best matched by the $\beta \approx 2.5$ curve. Both of these values match the prediction above, that $\beta=p+n$ with $n$ and $p$ independently determined from the data. These considerations imply that the 


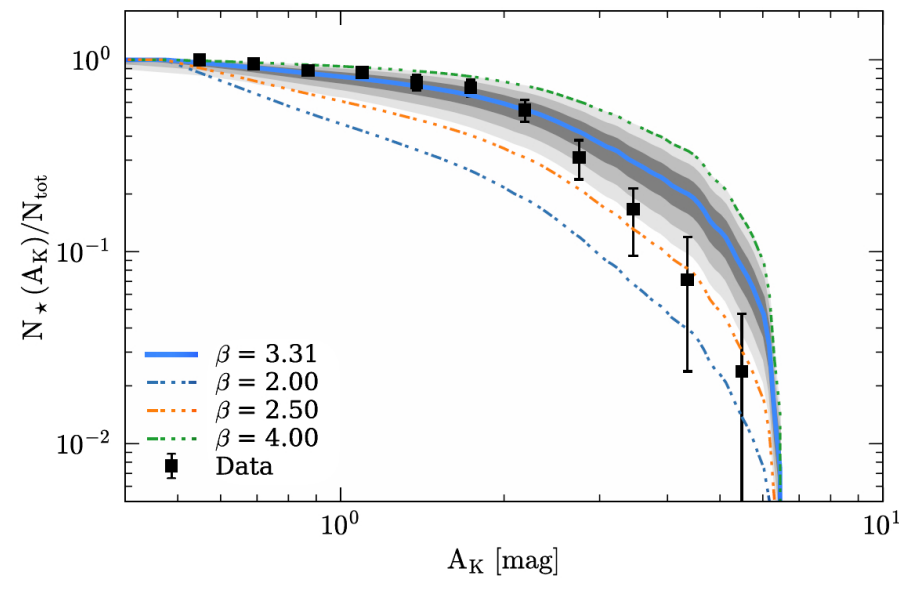

Fig. 12. Predicted distribution of protostars (solid line) calculated using the power-law relation, $\Sigma_{*}=0.36 A_{K}^{3.31}$, derived from the MCMC analysis here along with the measured cloud PDF. The gray shaded regions represent the 1,2 , and $3 \sigma$ confidence levels. The other lines show the predicted relations for different power-laws.

Schmidt relation in the CMC is not described by a single powerlaw that rises with extinction but instead is a somewhat more complex function, more steeply rising in the outer regions of the cloud than in the inner high extinction regions. A qualitatively similar behavior is observed in the cumulative distributions of protostars in the Orion A, Orion B and Perseus clouds and may be a general property of the internal Schmidt relation within a GMC. However, we note that the power-law indicies, $\beta$ and $n$, for the $\mathrm{CMC}$ are both greater than the corresponding indicies of the other three sources, which are very similar to each other. Yet the value of the index $p$ is essentially the same for all four sources. This may imply a certain similarity of $\operatorname{PDF}_{*}\left(A_{K}\right)$ between the local GMCs. A universal protostellar pdf would have interesting consequences for star formation theory. For example, Eq. (9) would then suggest that cloud-to-cloud variations in the slope of the Schmidt relation are primarily driven by variations in the slope of the cloud column density pdf.

\section{Conclusions}

We have constructed high-resolution, high dynamic range dust column-density and temperature maps of the California Molecular Cloud. The maps were derived by fitting Herschel fluxes in each map pixel with a modified blackbody to derive the dust opacities and effective (i.e., line-of-sight weighted) temperatures. The opacities were calibrated at low extinction by 2 MASS NIR extinction measurements to produce final maps of dust column density expressed as $A_{K}$. The column-density maps span a high dynamic range covering $0.04 \mathrm{mag}<A_{K}<11 \mathrm{mag}$, or $6.7 \times 10^{20} \mathrm{~cm}^{-2}<N<1.8 \times 10^{23} \mathrm{~cm}^{-2}$, a considerably larger range than measured in previous NIR extinction maps. This enables us to investigate cloud structure and star formation to much greater depths in the cloud than previously possible.

We used these data to determine the ratio of the $2.2 \mu \mathrm{m}$ extinction coefficient to the $850 \mu \mathrm{m}$ opacity and found the value $(\approx 3600)$ to be close to that $(3500)$ found in a similar study of the Orion B cloud but higher than that $(\approx 2500)$ characterizing the Orion A cloud, indicating that variations in the fundamental optical properties of dust may exist between local clouds.

We find that the column density pdf of the cloud can be well described over a large range of extinction $\left(0.35 \lesssim A_{K} \lesssim 11 \mathrm{mag}\right)$ by a simple power law (i.e., $\operatorname{PDF}_{N}\left(A_{K}\right) \propto A_{K}^{-n}$ ) with an index $(n=4.0 \pm 0.1)$ that represents a steeper decline with $A_{K}$ than found $(n \approx 3)$ in similar Herschel-Planck studies of the Orion A, Orion B, and Perseus clouds.

We re-examined existing catalogs of YSOs in the cloud and produced a merged catalog with slightly revised classifications for the known YSOs. Using only the protostellar population of the cloud and our Herschel extinction maps we investigated the Schmidt relation within the cloud. If we assumed that this relation is given by a simple power-law that is, $\Sigma_{*} \propto \Sigma_{\text {gas }}^{\beta}$, we found $\beta=3.31 \pm 0.23$, a slope that is significantly steeper than that $(\approx 2)$ found in other local GMCs. However modeling the cumulative distribution of protostars in the cloud indicated that $\Sigma_{*}$ is not a simple power-law function. Instead it is better described by two power-laws. At low extinction it is a rapidly rising power-law with an index of 3.3, while at higher extinctions it is characterized by a more slowly rising power-law with an index $\approx 2.5$.

We showed that $\Sigma_{*}$ is directly proportional to the ratio of the protostellar pdf, $\operatorname{PDF}_{*}\left(A_{K}\right)$, and $\operatorname{PDF}_{N}\left(A_{K}\right)$ and that if $\Sigma_{*}$ and $\mathrm{PDF}_{N}$ are simple power-law functions, $\mathrm{PDF}_{*}\left(A_{K}\right)$ must be a simple power-law as well, that is, $\operatorname{PDF}_{*}\left(A_{K}\right) \propto A_{K}^{p}$ where $p=\beta-n$.

We used the cumulative distribution of protostars to constrain the functional form of $\operatorname{PDF}_{*}\left(A_{K}\right)$. We found that it is not well described by a single power-law. At high extinctions $\operatorname{PDF}_{*}\left(A_{K}\right)$ is a declining power-law with $p \approx-1.4$. Between $0.5 \lesssim A_{K} \lesssim 2.5 \mathrm{mag}$ the function is characterized by a shallower power-law with $p \approx-0.5$. Below 0.5 mag it appears to be truncated and must fall steeply with decreasing extinction. Its behavior closely mirrors that of $\beta$, exactly as expected if $\mathrm{PDF}_{N}$ is well described by a single power-law function. Furthermore, our observations tentatively suggest that $\operatorname{PDF}_{*}\left(A_{K}\right)$ does not vary significantly between local clouds. If so, the variation in $\beta$ between the CMC and other nearby GMCs is largely driven by differences in the slope, $n$, of the column density pdfs of these clouds. However because the magnitude of the spectral index, $n$, of the cloud pdf is greater than that of the Schmidt relation, $\beta$, the size of the protostellar population and thus the global SFR in the cloud is largely controlled by the cloud's structure (i.e., $\operatorname{PDF}_{N}\left(A_{K}\right)$ ).

Finally, our observations of the CMC have provided valuable insights into the star formation process by adding to the evidence that cloud structure is critical to setting the level of star formation in a cloud. However, we have not explained why the CMC has the structure it does. Although this was likely inherited from its formation, we not do not know whether the $\mathrm{CMC}$ will remain a sleeping giant or awaken and evolve its structure to resemble the Orion clouds with considerably more high extinction material and the corresponding increased levels of star formation.

Acknowledgements. We thank Jan Forbrich for informative discussions. Based on observations obtained with Planck (http://www.esa.int/Planck), an ESA science mission with instruments and contributions directly funded by ESA Member States, NASA, and Canada. J. Alves acknowledges support from the Faculty of the European Space Astronomy Centre (ESAC) and J. Lewis acknowledges support from NSF GRFP grant DGE1144152.

\section{References}

André, P., Men'shchikov, A., Bontemps, S., et al. 2010, A\&A, 518, L102 Bohlin, R. C., Savage, B. D., \& Drake, J. F. 1978, ApJ, 224, 132 Broekhoven-Fiene, H., Matthews, B. C., Harvey, P. M., et al. 2014, ApJ, 786, 37 Evans, II, N. J., Heiderman, A., \& Vutisalchavakul, N. 2014, ApJ, 782, 114 Foreman-Mackey, D., Hogg, D. W., Lang, D., \& Goodman, J. 2013, PASP, 125, 306 
Griffin, M. J., Abergel, A., Abreu, A., et al. 2010, A\&A, 518, L3

Harvey, P. M., Fallscheer, C., Ginsburg, A., et al. 2013, ApJ, 764, 133

Heiderman, A., Evans, II, N. J., Allen, L. E., Huard, T., \& Heyer, M. 2010, ApJ, 723,1019

Jackson, J. M., Rathborne, J. M., Foster, J. B., et al. 2013, PASA, 30, e057 Kainulainen, J., Beuther, H., Henning, T., \& Plume, R. 2009, A\&A, 508, L35

Lada, C. J., Lombardi, M., \& Alves, J. F. 2009, ApJ, 703, 52

Lada, C. J., Lombardi, M., \& Alves, J. F. 2010, ApJ, 724, 687

Lada, C. J., Forbrich, J., Lombardi, M., \& Alves, J. F. 2012, ApJ, 745, 190

Lada, C. J., Lombardi, M., Roman-Zuniga, C., Forbrich, J., \& Alves, J. F. 2013, ApJ, 778, 133

Lewis, J. A., \& Lada, C. J. 2016, ApJ, 825, 1

Lombardi, M. 2009, A\&A, 493, 735

Lombardi, M., Lada, C. J., \& Alves, J. 2010, A\&A, 512, A67
Lombardi, M., Lada, C. J., \& Alves, J. 2013, A\&A, 559, A90

Lombardi, M., Bouy, H., Alves, J., \& Lada, C. J. 2014, A\&A, 566, A45

Lombardi, M., Alves, J., \& Lada, C. J. 2015, A\&A, 576, L1

Ott, S. 2010, in Astronomical Data Analysis Software and Systems XIX, Proc. Conf. held October 4-8, 139

Planck Collaboration XI. 2014, A\&A, 571, A11

Rieke, G. H., \& Lebofsky, M. J. 1985, ApJ, 288, 618

Robitaille, T. P., Whitney, B. A., Indebetouw, R., Wood, K., \& Denzmore, P. 2006, ApJS, 167, 256

Schmidt, M. 1959, ApJ, 129, 243

Vutisalchavakul, N., Evans, II, N. J., \& Heyer, M. 2016, ApJ, 831, 73

Wu, J., Evans, II, N. J., Gao, Y., et al. 2005, ApJ, 635, L173

Zari, E., Lombardi, M., Alves, J., Lada, C. J., \& Bouy, H. 2016, A\&A, 587, A106 


\section{Appendix A: The YSO catalog for the California Molecular Cloud}

The two most extensive catalogs of YSOs in the CMC are based on Spitzer, Herschel and WISE data (Harvey et al. 2013; Broekhoven-Fiene et al. 2014). Broekhoven-Fiene et al. selected Spitzer YSOs using color-color and color-magnitude diagrams and then used Herschel, Spitzer and WISE observations to construct SEDs with sufficient wavelength coverage to provide suitable classifications of the objects. The $2-24 \mu \mathrm{m}$ SED slope was employed to obtain their YSO classifications, producing 58 protostellar objects including objects classified as flat-spectrum sources. Harvey et al. used SED slopes from 3.6-160 $\mu \mathrm{m}$ to classify the Herschel sources in the CMC, and used the bolometric temperature $\left(T_{\text {bol }}\right)$ over the same range to separate Class $0\left(T_{\text {bol }} \leq 50 \mathrm{~K}\right)$ and Class I $\left(50 \mathrm{~K} \leq T_{\text {bol }} \leq 70 \mathrm{~K}\right)$ sources. They found 49 sources with protostellar like SEDs Because the two analyses are independent, there is some disagreement between the catalogs in classifications of the same sources. By combining both data sets we create better, more complete, SEDs to use for source classification. We re-examined the source classifications following the methodology of Lewis \& Lada (2016). We first ensured proper matching by requiring that every object Harvey et al. matched to the Broekhoven-Fiene et al. catalog (see Harvey et al. 2013, Table 2) is matched in our merged list, and we resolve conflicts by examining every match by eye. We fitted the source SEDs using the SED models developed by Robitaille et al. (2006) to obtain estimates of the quantities $M_{\star}$, the mass of the central star, $\dot{M}$ the mass accretion/infall rate, $M_{\text {env }}$, the mass of the protostellar, infalling envelope, and $M_{\text {disk}}$, the mass of any circumstellar disk. We then assign classifications using the following criteria:

- P: protostar (Class 0/I), $\dot{M} / M_{\star} \geq 10^{-6}$ and $M_{\text {env }} / M_{\star} \geq 0.05$; - D: disk (Class II), $\dot{M} / M_{\star}<10^{-6}$ and $M_{\text {disk }} / M_{\star} \geq 10^{-6}$; - S: star (Class III) $\dot{M} / M_{\star}<10^{-6}$ and $M_{\text {disk }} / M_{\star}<10^{-6}$.

In this manner we determined that 42 sources in the merged list of Table A.1 were protostars. We note that the difference between this estimate and that of Broekhoven-Fiene et al. is primarily due to our assignment of a disk classification for most of the flat spectrum sources in the Broekhoven-Fiene et al. list. We further note that sources 1-3 are outside Herschel footprint. Their extinctions are derived from Planck. One of these we classify as a protostar but it was not included in any of our subsequent analysis. 
Table A.1. Young stellar objects in the California Molecular Cloud.

\begin{tabular}{|c|c|c|c|c|c|c|c|c|}
\hline \multirow[t]{2}{*}{ ID } & \multirow[t]{2}{*}{ RA (J2000) } & \multirow[t]{2}{*}{ Dec (J2000) } & \multirow[t]{2}{*}{$A_{K}$ (error) } & \multicolumn{2}{|c|}{ Broekhoven-Fiene et al. } & \multicolumn{2}{|c|}{ Harvey et al. } & \multirow[t]{2}{*}{ Class } \\
\hline & & & & ID & Class & ID & Class & \\
\hline $1 *$ & 040124.55 & 410149.00 & $0.819(0.007)$ & 1 & $\mathrm{I}$ & 0 & - & $\mathrm{P}$ \\
\hline $2 *$ & 040134.36 & 411143.00 & $0.934(0.020)$ & 2 & II & 0 & - & $\mathrm{D}$ \\
\hline $3^{*}$ & 040229.75 & 404241.90 & $0.313(0.008)$ & 139 & II & 0 & - & $\mathrm{D}$ \\
\hline 4 & 040902.00 & 401913.10 & $1.190(0.029)$ & 140 & I & 1 & I & $\mathrm{P}$ \\
\hline 5 & 040954.71 & 400639.89 & $4.096(0.054)$ & - & - & 2 & I & $\mathrm{P}$ \\
\hline 6 & 041000.64 & 400236.10 & $2.857(0.054)$ & 3 & II & - & - & $\mathrm{D}$ \\
\hline 7 & 041002.63 & 400248.20 & $3.051(0.050)$ & 4 & I & 3 & 0 & $\mathrm{P}$ \\
\hline 8 & 041003.43 & 390449.50 & $0.338(0.004)$ & 141 & III & - & - & $\mathrm{S}$ \\
\hline 9 & 041004.53 & 400237.50 & $2.415(0.053)$ & - & - & 4 & 0 & $\mathrm{P}$ \\
\hline 10 & 041005.62 & 400238.60 & $3.146(0.062)$ & 5 & II & 5 & 0 & $\mathrm{D}$ \\
\hline 11 & 041007.08 & 400234.58 & $2.717(0.068)$ & - & - & 6 & 0 & $\mathrm{P}$ \\
\hline 12 & 041008.41 & 400224.40 & $2.621(0.076)$ & 6 & I & 7 & I & $\mathrm{P}$ \\
\hline 13 & 041011.16 & 400126.20 & $2.390(0.071)$ & 7 & I & 8 & 0 & $\mathrm{P}$ \\
\hline 14 & 041024.41 & 380522.70 & $0.435(0.008)$ & 142 & II & - & - & D \\
\hline 15 & 041040.51 & 380500.40 & $1.339(0.034)$ & 8 & II & - & - & D \\
\hline 16 & 041041.09 & 380754.50 & $1.917(0.090)$ & 10 & I & 9 & I & $\mathrm{P}$ \\
\hline 17 & 041041.63 & 380805.80 & $3.277(0.118)$ & 9 & II & - & - & D \\
\hline 18 & 041042.11 & 380559.90 & $1.682(0.017)$ & 11 & III & - & - & $\mathrm{S}$ \\
\hline 19 & 041047.61 & 380333.80 & $0.383(0.010)$ & 12 & II & - & - & D \\
\hline 20 & 041049.16 & 380445.80 & $2.714(0.054)$ & 13 & II & 10 & $\mathrm{~F}$ & D \\
\hline 21 & 041208.47 & 380146.60 & $0.341(0.005)$ & 143 & III & - & - & $\mathrm{S}$ \\
\hline 22 & 041240.54 & 381426.81 & $0.549(0.007)$ & - & - & 11 & $\mathrm{I} / 0$ & $\mathrm{P}$ \\
\hline 23 & 041257.64 & 391418.30 & $0.293(0.005)$ & 144 & III & - & - & $\mathrm{S}$ \\
\hline 24 & 041344.57 & 390435.70 & $0.414(0.005)$ & 145 & III & - & - & $\mathrm{S}$ \\
\hline 25 & 041511.20 & 383957.10 & $0.341(0.005)$ & 146 & II & - & - & D \\
\hline 26 & 041554.05 & 383413.10 & $0.396(0.005)$ & 147 & III & - & - & $S$ \\
\hline 27 & 041705.93 & 372218.70 & $0.208(0.005)$ & 148 & III & - & - & $\mathrm{S}$ \\
\hline 28 & 041944.67 & 381121.90 & $0.501(0.006)$ & 14 & $\mathrm{~F}$ & - & - & D \\
\hline 29 & 042052.46 & 380635.80 & $0.604(0.007)$ & 15 & III & - & - & $\mathrm{S}$ \\
\hline 30 & 042137.95 & 373441.80 & $3.497(0.062)$ & 16 & II & 12 & $\mathrm{~F}$ & $\mathrm{D}$ \\
\hline 31 & 042138.08 & 373540.90 & $3.167(0.050)$ & 17 & III & - & - & $\mathrm{S}$ \\
\hline 32 & 042140.80 & 373359.00 & $4.874(0.080)$ & 18 & I & 13 & I & $\mathrm{P}$ \\
\hline 33 & 042305.46 & 380736.90 & $0.271(0.005)$ & 149 & III & - & - & $\mathrm{S}$ \\
\hline 34 & 042449.34 & 371646.40 & $1.128(0.008)$ & 19 & III & - & - & $\mathrm{S}$ \\
\hline 35 & 042459.04 & 371752.91 & $1.307(0.020)$ & - & - & 14 & I & $\mathrm{P}$ \\
\hline 36 & 042507.83 & 371519.30 & $2.052(0.053)$ & - & - & 15 & 0 & $\mathrm{P}$ \\
\hline 37 & 042538.48 & 370701.20 & $6.295(0.068)$ & 20 & I & 16 & $\mathrm{I} / 0$ & $\mathrm{P}$ \\
\hline 38 & 042539.79 & 370708.20 & $6.452(0.051)$ & 21 & $\mathrm{~F}$ & 17 & II & D \\
\hline 39 & 042713.74 & 362710.70 & $0.428(0.003)$ & 150 & II & - & - & $\mathrm{D}$ \\
\hline 40 & 042750.80 & 363126.40 & $0.523(0.003)$ & 22 & II & - & - & $\mathrm{D}$ \\
\hline 41 & 042758.26 & 363326.50 & $0.546(0.003)$ & 23 & II & - & - & D \\
\hline 42 & 042802.89 & 364058.60 & $0.802(0.004)$ & 24 & II & - & - & D \\
\hline 43 & 042815.15 & 363028.60 & $1.262(0.004)$ & 25 & $\mathrm{~F}$ & 18 & $\mathrm{~F}$ & D \\
\hline 44 & 042821.16 & 362447.80 & $0.753(0.003)$ & 26 & II & - & - & D \\
\hline 45 & 042821.36 & 363021.50 & $0.919(0.003)$ & 27 & II & - & - & $\mathrm{D}$ \\
\hline 46 & 042835.09 & 362506.40 & $1.961(0.020)$ & 28 & I & 19 & I & $\mathrm{P}$ \\
\hline 47 & 042837.89 & 362455.30 & $1.757(0.017)$ & 29 & II & 20 & $\mathrm{~F}$ & D \\
\hline 48 & 042838.56 & 362528.90 & $2.815(0.021)$ & 30 & I & 21 & I & $\mathrm{P}$ \\
\hline 49 & 042843.35 & 362511.70 & $0.887(0.005)$ & 31 & II & - & - & D \\
\hline 50 & 042843.67 & 362839.30 & $1.801(0.023)$ & 32 & I & 22 & $\mathrm{I} / 0$ & $\mathrm{P}$ \\
\hline 51 & 042844.43 & 362445.60 & $0.758(0.004)$ & 33 & $\mathrm{~F}$ & - & - & D \\
\hline 52 & 042849.58 & 362910.70 & $1.165(0.004)$ & 34 & I & - & - & D \\
\hline 53 & 042855.30 & 363122.50 & $1.965(0.035)$ & 35 & I & 23 & I & $\mathrm{P}$ \\
\hline 54 & 042855.56 & 352446.00 & $0.305(0.004)$ & 151 & II & - & - & D \\
\hline 55 & 042859.11 & 362311.20 & $0.442(0.003)$ & 36 & II & - & - & D \\
\hline 56 & 042911.53 & 350449.50 & $0.233(0.005)$ & 152 & II & - & - & $\mathrm{D}$ \\
\hline 57 & 042914.38 & 351524.50 & $0.363(0.004)$ & 153 & II & - & - & D \\
\hline 58 & 042939.01 & 351610.50 & $0.459(0.003)$ & 37 & II & - & - & $\mathrm{D}$ \\
\hline 59 & 042940.01 & 352108.90 & $0.696(0.006)$ & 38 & I & - & - & $\mathrm{P}$ \\
\hline
\end{tabular}


Table A.1. continued.

\begin{tabular}{|c|c|c|c|c|c|c|c|c|}
\hline \multirow[t]{2}{*}{ ID } & \multirow[t]{2}{*}{ RA $(J 2000)$} & \multirow[t]{2}{*}{ Dec (J2000) } & \multirow[t]{2}{*}{$A_{K}$ (error) } & \multicolumn{2}{|c|}{ Broekhoven-Fiene et al. } & \multicolumn{2}{|c|}{ Harvey et al. } & \multirow[t]{2}{*}{ Class } \\
\hline & & & & & Class & & Class & \\
\hline 60 & 042943.58 & 351338.60 & $0.646(0.004)$ & 39 & II & - & - & $\mathrm{D}$ \\
\hline 61 & 042944.21 & 351230.00 & $0.612(0.005)$ & 40 & $\mathrm{~F}$ & - & - & $\mathrm{D}$ \\
\hline 62 & 042946.28 & 361923.50 & $0.423(0.003)$ & 154 & $\mathrm{~F}$ & - & - & $\mathrm{D}$ \\
\hline 63 & 042947.28 & 351019.20 & $0.406(0.003)$ & 41 & II & - & - & $\mathrm{D}$ \\
\hline 64 & 042947.42 & 351133.50 & $0.605(0.006)$ & 42 & II & - & - & $\mathrm{D}$ \\
\hline 65 & 042948.54 & 351212.50 & $0.671(0.004)$ & 43 & II & - & - & $\mathrm{D}$ \\
\hline 66 & 042949.21 & 351422.70 & $1.553(0.019)$ & 44 & $\mathrm{~F}$ & - & - & $\mathrm{D}$ \\
\hline 67 & 042949.61 & 351443.80 & $2.186(0.025)$ & 45 & II & - & - & $\mathrm{D}$ \\
\hline 68 & 042950.17 & 351444.50 & $2.186(0.025)$ & 136 & II & - & - & $\mathrm{D}$ \\
\hline 69 & 042950.84 & 351557.90 & $2.249(0.013)$ & 46 & $\mathrm{~F}$ & - & - & $\mathrm{D}$ \\
\hline 70 & 042951.01 & 351547.50 & $2.249(0.013)$ & 47 & I & - & - & $\mathrm{P}$ \\
\hline 71 & 042952.54 & 352223.60 & $0.292(0.003)$ & 155 & III & - & - & $\mathrm{S}$ \\
\hline 72 & 042953.46 & 351548.50 & $2.108(0.013)$ & 48 & $\mathrm{~F}$ & - & - & $\mathrm{D}$ \\
\hline 73 & 042954.15 & 351021.60 & $0.457(0.006)$ & 49 & $\mathrm{~F}$ & - & - & $\mathrm{D}$ \\
\hline 74 & 042954.18 & 361157.30 & $0.985(0.006)$ & 156 & $\mathrm{~F}$ & 24 & II & $\mathrm{D}$ \\
\hline 75 & 042954.79 & 351802.50 & $0.780(0.006)$ & 50 & II & 25 & $\mathrm{~F}$ & $\mathrm{D}$ \\
\hline 76 & 042956.27 & 351742.90 & $0.760(0.009)$ & 51 & I & - & - & $\mathrm{P}$ \\
\hline 77 & 042959.19 & 361016.10 & $0.773(0.006)$ & 157 & II & 26 & II & $\mathrm{D}$ \\
\hline 78 & 042959.76 & 351334.20 & $0.788(0.006)$ & 52 & II & - & - & $\mathrm{D}$ \\
\hline 79 & 043000.16 & 360322.70 & $0.531(0.004)$ & 53 & II & - & - & D \\
\hline 80 & 043001.14 & 351724.60 & $0.333(0.002)$ & 54 & III & - & - & $\mathrm{S}$ \\
\hline 81 & 043001.52 & 360733.30 & $0.449(0.003)$ & 158 & II & - & - & $\mathrm{D}$ \\
\hline 82 & 043001.88 & 353814.70 & $0.350(0.005)$ & 159 & II & - & - & $\mathrm{D}$ \\
\hline 83 & 043002.63 & 351514.30 & $0.831(0.006)$ & 55 & II & - & - & $\mathrm{D}$ \\
\hline 84 & 043003.63 & 351420.10 & $2.331(0.016)$ & 56 & I & - & - & $\mathrm{P}$ \\
\hline 85 & 043004.23 & 350945.90 & $0.523(0.005)$ & 57 & II & - & - & D \\
\hline 86 & 043004.25 & 352223.80 & $0.336(0.002)$ & 58 & II & - & - & $\mathrm{D}$ \\
\hline 87 & 043007.43 & 351457.90 & $0.659(0.005)$ & 59 & II & - & - & $\mathrm{D}$ \\
\hline 88 & 043007.73 & 351548.40 & $0.429(0.002)$ & 60 & II & - & - & $\mathrm{D}$ \\
\hline 89 & 043008.25 & 351410.00 & $1.528(0.009)$ & 61 & I & - & - & $\mathrm{P}$ \\
\hline 90 & 043008.74 & 351437.50 & $1.434(0.010)$ & 62 & II & - & - & D \\
\hline 91 & 043009.51 & 351440.30 & $1.101(0.006)$ & 63 & I & - & - & $\mathrm{P}$ \\
\hline 92 & 043009.80 & 354035.50 & $0.447(0.005)$ & 64 & II & - & - & $\mathrm{D}$ \\
\hline 93 & 043009.80 & 361335.40 & $0.444(0.003)$ & 160 & II & - & - & D \\
\hline 94 & 043009.86 & 351416.30 & $1.528(0.010)$ & 137 & II & - & - & $\mathrm{D}$ \\
\hline 95 & 043009.91 & 351553.90 & $0.420(0.003)$ & 65 & II & - & - & $\mathrm{D}$ \\
\hline 96 & 043012.34 & 350934.60 & $1.531(0.015)$ & 66 & II & - & - & D \\
\hline 97 & 043013.09 & 351358.60 & $1.165(0.006)$ & 67 & II & - & - & $\mathrm{D}$ \\
\hline 98 & 043014.53 & 351332.60 & $1.315(0.008)$ & 68 & II & - & - & D \\
\hline 99 & 043014.74 & 352014.30 & $0.583(0.004)$ & 69 & II & - & - & $\mathrm{D}$ \\
\hline 100 & 043014.95 & 360008.50 & $2.047(0.028)$ & 70 & I & 27 & I & $\mathrm{D}$ \\
\hline 101 & 043015.21 & 351639.80 & $1.067(0.012)$ & 138 & $\mathrm{~F}$ & - & - & D \\
\hline 102 & 043015.76 & 355657.80 & $0.753(0.020)$ & 71 & I & 28 & II & $\mathrm{D}$ \\
\hline 103 & 043016.27 & 354242.90 & $0.424(0.005)$ & 72 & II & - & - & D \\
\hline 104 & 043017.84 & 360326.60 & $0.602(0.005)$ & 73 & III & - & - & $\mathrm{S}$ \\
\hline 105 & 043018.08 & 354538.90 & $0.546(0.008)$ & 74 & II & - & - & $\mathrm{D}$ \\
\hline 106 & 043018.99 & 354212.00 & $0.479(0.006)$ & 75 & II & - & - & D \\
\hline 107 & 043019.59 & 350821.60 & $1.220(0.015)$ & 76 & F & - & - & $\mathrm{D}$ \\
\hline 108 & 043022.19 & 360435.90 & $0.857(0.008)$ & 77 & II & - & - & $\mathrm{D}$ \\
\hline 109 & 043022.68 & 351908.10 & $0.617(0.007)$ & 78 & II & - & - & D \\
\hline 110 & 043023.82 & 352112.30 & $0.740(0.007)$ & 79 & I & - & - & $\mathrm{P}$ \\
\hline 111 & 043024.33 & 345916.50 & $0.440(0.006)$ & 80 & III & - & - & $\mathrm{S}$ \\
\hline 112 & 043024.68 & 354520.60 & $1.221(0.028)$ & 81 & I & 29 & I & $\mathrm{P}$ \\
\hline 113 & 043025.03 & 354317.90 & $1.115(0.012)$ & 82 & II & - & - & $\mathrm{D}$ \\
\hline 114 & 043025.89 & 354811.30 & $1.507(0.019)$ & 83 & II & - & - & $\mathrm{D}$ \\
\hline 115 & 043027.02 & 352028.40 & $0.811(0.008)$ & 84 & II & - & - & $\mathrm{D}$ \\
\hline 116 & 043027.04 & 354550.50 & $2.062(0.023)$ & 85 & F & 30 & I & $\mathrm{P}$ \\
\hline 117 & 043027.41 & 350917.80 & $1.919(0.077)$ & 86 & I & 31 & I & $\mathrm{P}$ \\
\hline 118 & 043027.75 & 354615.00 & $2.629(0.022)$ & 87 & $\mathrm{~F}$ & 32 & II & $\mathrm{D}$ \\
\hline
\end{tabular}


Table A.1. continued.

\begin{tabular}{|c|c|c|c|c|c|c|c|c|}
\hline \multirow[t]{2}{*}{ ID } & \multirow[t]{2}{*}{ RA (J2000) } & \multirow[t]{2}{*}{ Dec (J2000) } & \multirow[t]{2}{*}{$A_{K}$ (error) } & \multicolumn{2}{|c|}{ Broekhoven-Fiene et al. } & \multicolumn{2}{|c|}{ Harvey et al. } & \multirow[t]{2}{*}{ Class } \\
\hline & & & & & Class & & Class & \\
\hline 119 & 043028.09 & 350916.40 & $2.970(0.101)$ & 88 & $\mathrm{I}$ & - & - & $\mathrm{P}$ \\
\hline 120 & 043028.42 & 353241.90 & $0.428(0.005)$ & 89 & II & - & - & $\mathrm{D}$ \\
\hline 121 & 043028.44 & 354917.60 & $2.590(0.015)$ & 90 & $\mathrm{~F}$ & - & - & $\mathrm{D}$ \\
\hline 122 & 043028.61 & 354740.70 & $1.798(0.015)$ & 91 & II & 33 & II & $\mathrm{D}$ \\
\hline 123 & 043028.71 & 354749.80 & $1.826(0.018)$ & 92 & II & - & - & $\mathrm{D}$ \\
\hline 124 & 043028.98 & 350754.00 & $0.769(0.009)$ & 93 & II & - & - & $\mathrm{D}$ \\
\hline 125 & 043029.61 & 352717.20 & $0.965(0.010)$ & 94 & II & - & - & $\mathrm{D}$ \\
\hline 126 & 043029.66 & 350639.00 & $1.102(0.010)$ & 95 & II & - & - & $\mathrm{D}$ \\
\hline 127 & 043030.14 & 350639.20 & $1.154(0.011)$ & 96 & II & 34 & II & $\mathrm{D}$ \\
\hline 128 & 043030.28 & 352104.00 & $0.581(0.006)$ & 97 & II & - & - & $\mathrm{D}$ \\
\hline 129 & 043030.43 & 351833.70 & $0.335(0.003)$ & 98 & II & - & - & $\mathrm{D}$ \\
\hline 130 & 043030.51 & 351744.70 & $0.383(0.004)$ & 99 & II & - & - & $\mathrm{D}$ \\
\hline 131 & 043030.56 & 355144.00 & $3.344(0.053)$ & 100 & I & 35 & I & $\mathrm{P}$ \\
\hline 132 & 043031.58 & 354513.70 & $3.073(0.039)$ & 101 & $\mathrm{~F}$ & 36 & II & $\mathrm{D}$ \\
\hline 133 & 043032.35 & 353613.40 & $1.062(0.016)$ & 102 & II & 37 & $\mathrm{~F}$ & $\mathrm{P}$ \\
\hline 134 & 043036.80 & 355436.20 & $4.275(0.132)$ & 103 & I & 38 & I & $\mathrm{P}$ \\
\hline 135 & 043037.40 & 360018.00 & $0.711(0.009)$ & 104 & II & - & - & $\mathrm{D}$ \\
\hline 136 & 043037.51 & 351348.60 & $0.460(0.005)$ & 105 & II & - & - & $\mathrm{D}$ \\
\hline 137 & 043037.51 & 355031.70 & $4.321(0.101)$ & 106 & II & 39 & $\mathrm{~F}$ & $\mathrm{D}$ \\
\hline 138 & 043037.89 & 355101.40 & $3.383(0.080)$ & 107 & I & 40 & 0 & $\mathrm{P}$ \\
\hline 139 & 043038.26 & 354959.30 & $2.342(0.077)$ & 108 & II & 41 & 0 & $\mathrm{P}$ \\
\hline 140 & 043038.38 & 355022.60 & $3.720(0.092)$ & - & - & 42 & 0 & $\mathrm{P}$ \\
\hline 141 & 043038.65 & 355439.10 & $4.404(0.162)$ & 109 & F & 43 & $\mathrm{~F}$ & D \\
\hline 142 & 043039.12 & 354449.80 & $0.726(0.008)$ & 110 & II & - & - & $\mathrm{D}$ \\
\hline 143 & 043039.16 & 355203.80 & $2.276(0.040)$ & 111 & F & 44 & $\mathrm{~F}$ & $\mathrm{P}$ \\
\hline 144 & 043039.31 & 355200.70 & $2.647(0.037)$ & 112 & $\mathrm{~F}$ & - & - & D \\
\hline 145 & 043039.56 & 351806.90 & $0.337(0.004)$ & 113 & II & - & - & $\mathrm{D}$ \\
\hline 146 & 043039.58 & 351112.80 & $0.403(0.004)$ & 114 & II & - & - & $\mathrm{D}$ \\
\hline 147 & 043040.05 & 354210.30 & $0.363(0.006)$ & 115 & III & - & - & $\mathrm{S}$ \\
\hline 148 & 043040.14 & 353134.10 & $1.497(0.032)$ & 116 & II & - & - & D \\
\hline 149 & 043041.16 & 352941.00 & $4.681(0.069)$ & 117 & I & 45 & I & $\mathrm{P}$ \\
\hline 150 & 043044.23 & 355951.10 & $2.782(0.061)$ & 118 & I & 46 & $\mathrm{~F}$ & $\mathrm{D}$ \\
\hline 151 & 043044.69 & 351052.10 & $0.370(0.005)$ & 119 & II & - & - & $\mathrm{D}$ \\
\hline 152 & 043045.58 & 345808.00 & $0.997(0.017)$ & 120 & II & - & - & D \\
\hline 153 & 043046.25 & 345856.20 & $2.743(0.096)$ & 121 & I & 47 & 0 & $\mathrm{P}$ \\
\hline 154 & 043047.23 & 350743.20 & $0.745(0.008)$ & 122 & II & 48 & II & $\mathrm{D}$ \\
\hline 155 & 043047.57 & 345824.20 & $3.860(0.143)$ & 123 & II & - & - & D \\
\hline 156 & 043047.90 & 345837.31 & $3.860(0.143)$ & - & - & 49 & 0 & $\mathrm{P}$ \\
\hline 157 & 043048.52 & 353753.70 & $2.480(0.051)$ & 124 & I & 50 & I & $\mathrm{P}$ \\
\hline 158 & 043048.61 & 345853.50 & $5.167(0.168)$ & 125 & I & 51 & $\mathrm{~F}$ & $\mathrm{~S}$ \\
\hline 159 & 043049.22 & 345610.30 & $1.025(0.012)$ & 126 & I & 52 & $\mathrm{~F}$ & $\mathrm{D}$ \\
\hline 160 & 043049.33 & 345046.00 & $0.585(0.009)$ & 161 & II & - & - & D \\
\hline 161 & 043049.34 & 353641.90 & $0.808(0.011)$ & 127 & II & - & - & $\mathrm{D}$ \\
\hline 162 & 043049.48 & 345056.20 & $0.559(0.009)$ & 162 & II & - & - & D \\
\hline 163 & 043049.68 & 345727.70 & $0.860(0.021)$ & 128 & II & 53 & II & D \\
\hline 164 & 043050.57 & 353323.50 & $0.864(0.007)$ & 129 & II & - & - & $\mathrm{D}$ \\
\hline 165 & 043050.98 & 353554.80 & $0.516(0.007)$ & 130 & II & - & - & D \\
\hline 166 & 043052.08 & 345008.90 & $0.653(0.010)$ & 163 & F & 54 & $\mathrm{~F}$ & $\mathrm{D}$ \\
\hline 167 & 043053.50 & 345627.40 & $2.593(0.041)$ & 131 & I & 55 & I & $\mathrm{D}$ \\
\hline 168 & 043053.90 & 353011.00 & $1.097(0.017)$ & 132 & II & - & - & D \\
\hline 169 & 043055.01 & 353056.20 & $0.583(0.006)$ & 133 & II & - & - & $\mathrm{D}$ \\
\hline 170 & 043055.99 & 345647.80 & $2.140(0.028)$ & 134 & I & 56 & I & D \\
\hline 171 & 043056.61 & 353004.50 & $1.513(0.029)$ & 135 & I & 57 & I & $\mathrm{P}$ \\
\hline 172 & 043057.19 & 345353.59 & $2.799(0.058)$ & - & - & 58 & I & $\mathrm{P}$ \\
\hline 173 & 043114.67 & 355650.60 & $0.633(0.008)$ & - & - & 59 & I & $\mathrm{P}$ \\
\hline 174 & 043205.77 & 360637.50 & $0.359(0.005)$ & 164 & III & - & - & $\mathrm{S}$ \\
\hline 175 & 043254.31 & 360444.00 & $0.362(0.005)$ & 165 & II & - & - & D \\
\hline 176 & 043303.15 & 360204.50 & $0.360(0.006)$ & 166 & II & - & - & D \\
\hline 177 & 043453.15 & 362327.89 & $2.284(0.041)$ & - & - & 60 & II & $\mathrm{D}$ \\
\hline
\end{tabular}

